\title{
Toward an adequate mathematical model of mental space: conscious/unconscious dynamics on $m$-adic trees
}

\author{
Andrei Yu. Khrennikov \\ Center for Mathematical Modeling \\ in Physics and Cognitive Sciences, \\ University of Växjö, S-35195, Sweden \\ Email:Andrei.Khrennikov@msi.vxu.se
}

November 4, 2018

\begin{abstract}
We try to perform geometrization of cognitive science and psychology by representing information states of cognitive systems by points of mental space given by a hierarchic $m$-adic tree. Associations are represented by balls and ideas by collections of balls. We consider dynamics of ideas based on lifting of dynamics of mental points. We apply our dynamical model for modeling of flows of unconscious and conscious information in the human brain. In a series of models, Models 1-3, we consider cognitive systems with increasing complexity of psychological behavior determined by structure of flows of associations and ideas.

Keywords: Mental space, hierarchic encoding of mental information, madic trees and numbers, dynamical systems, conscious/unconscious flows of information. neuronal trees, psychoanalysis
\end{abstract}

\section{Introduction}

One of the sources of the extremely successful mathematical formalization of physics was creation of the adequate mathematical model of physical space, namely, the Cartesian product of real lines. This provides the possibility for "embedding" physical objects into a mathematical space. Coordinates of physical systems are given by points of this space. Rigid physical bodies are represented by geometric figures (cubes, balls,... ). By describing dynamics of coordinates, e.g., with the aid of differential equations, we can describe dynamics of bodies (from falling stones to Sputniks). 
In a series of works (Khrennikov, 1997, 1998a,b, 1999a,b, 2000a,b) there was advocated a similar approach to description of mental processes in cognitive sciences and psychology (and even information dynamics in genetics), see also Albeverio et al., 1999, and Dubischar et al, 1999. Similar to physics, the first step should be elaboration of a mathematical model of mental space. We understood well that this is a problem of huge complexity and it might take a few hundred years for creating an adequate mathematical model of mental space. We recall that it took three hundred years to create a mathematically rigorous model of real physical space. In previous papers critical arguments were presented against the real model of space as a possible candidate for a mental space. One of the main arguments was that the real continuum is a continuous infinitely divisible space. Such a picture of space is adequate to physical space (at least in classical physics), but mental space is not continuous: mind is not infinitely divisible! Another problem with the real continuum is that it is homogeneous: "all points of this space have equal rights." In opposition to such a homogeneity mental states have clearly expressed hierarchical structure, see for discussions: Hubel and Wiesel, 1962, Smythies, 1970, Clark, 1980, Amit, 1989, Bechtel and Abrahamsen, 1991, Khrennikov, 1997, 1998a,b, 1999a,b, 2000a, b; Albeverio et al., 1999; Dubischar et al, 1999, Voronkov, 2002a, b, Sergin, 2007, see also Bechterew, 1911, Damasio et al., 1989, Fuster, 1997, for corresponding medical evidence.

Therefore a model of mental space that we are looking for should be (at least) discontinuous and hierarchical. In mathematics there is a well known class of spaces with such features. These are $m$-adic trees (here $m$ is a natural number giving the number of branches of a tree at each vertex). It is interesting that such trees are nicely equipped: there is a well defined algebraic structure which gives the possibility to add, subtract, multiply, and for prime $m$ (so $m=2,3,5, \ldots, 1997,1999, \ldots$ ) even divide branches of such a tree. There is a natural topology on such trees encoding the hierarchic tree structure. This topology is based on a metric, so called ultrametric. Thus $m$ adic trees are not worse equipped than the real line. However, the equipment - algebra and topology - is very different from the real one.

We proposed (see Khrennikov, 1997, 1998a,b, 1999a,b, 2000a,b; Albeverio et al., 1999; Dubischar et al, 1999) to choose $m$-adic trees as possible models of mental space $X_{\text {mental }}$ : points of this space are branches of a tree. These are mental coordinates representing mental states. By using mental coordinates we able to embed into the space mental analogs of physical rigid bodies - associations and ideas. They are represented, respectively, by balls and collections of balls in the ultrametric mental space.

Mental states (represented by branches) are basic cognitive mental images. An association connects a number of cognitive mental images. Thus an association can be represented as a subset of the mental space. The crucial point is that in our model the associative connection of cognitive mental images is fundamentally hierarchical. Therefore an association is not an arbitrary collection of cognitive mental images (not an arbitrary set of mental 
points), but a hierarchically coupled collection. Since in our model the mental hierarchy is encoded by the topology of the mental space, it represents the associative coupling of cognitive mental images into balls. A larger ball couples together more cognitive mental images. Thus it is a more complex association (but it is a "fuzzy-association," it is not sharp). Decreasing of ball's radius induces decreasing of the complexity an association which is represented by this ball. An association becomes sharper. In the limit we obtain the ball of the zero radius. That is nothing else than a single mental point (the center of such a degenerated ball). This is a single cognitive mental image. This is the limiting case of an association: a cognitive mental image is "associated" with itself. We hope that such a limiting degeneration of an association into a mental image would not be misleading for readers.

Ideas are identified as collections of associations, something analogous to a coherent group of individuals in the biological analogy. The identification of the fundamental structure as a mental image allows a concrete dynamical model for ideas as collections of loosely bound associations. Association is kind of atom of cognition from which more complex ideas are build like molecules from atoms.

We mention also that a $p$-adic model (here $m=p$ is a prime number) of consciousness was (independently) proposed in Pitkänen, 1998. Pitkänen's approach was not based on encoding of mental hierarchy by $p$-adic numbers. It has a deeper relation to foundations of physics, especially the quantum one.

Recently $p$-adic information space was used for genetic models, see Dragovich et al., 2006, and Khrennikov, 2006a,b as well as Pitkänen, 2006. A new exciting domain of research is use of ultrametric methods in data-analysis from astrophysics and computer science to biology, see, e.g., Murtagh, 2004.

In papers, Khrennikov, 1997, 1998a,b, 1999a,b; Albeverio et al., 1999; Dubischar et al, 1999, we studied merely the dynamics of mental states mental images. We considered dynamical systems which work with mental states. There is a nonlinear relation between input and output mental states,

$$
x_{n+1}=f\left(x_{n}\right), x_{n} \in X_{\text {mental }} .
$$

The description of functioning of the human brain by dynamical systems (feedback processes) is a well established approach. The main difference between our approach and the conventional dynamical approach to cognition (see Ashby, 1952, van Gelder and Port, 1995, van Gelder, 1995, Strogatz, 1994, Eliasmith, 1996, Conte et al, 2006 - in the latter there was presented a dynamical model of cognition exhibiting nondeterministic features similar to those in quantum mechanics) is that in the conventional dynamical approach dynamical systems work in the real physical space of electric potentials and in our approach dynamical systems work in the $m$-adic mental space. There is also a similarity between our approach and the artificial intelligence approach, Chomsky, 1963, Churchland and Sejnovski, 1992.

In the present paper we study dynamics of mental analogs of physical rigid bodies - associations (balls in the mental metric space) and ideas (col- 
lections of balls). In spite of the fact that dynamics of associations and ideas can be in principle reduced to dynamics of mental points composing those "mental bodies", the those dynamics exhibits their own interesting properties which could not be seen on the level of pointwise dynamics.

We apply our dynamical model for modeling of flows of unconscious and conscious information in the human brain 1

In series of models, Models 1-3, we consider cognitive systems with increasing complexity of psychological behavior determined by structure of flows of associations and ideas. Using this basic conceptual repertoire an increasingly refined cognitive model is developed starting from an animal like individual whose sexual behavior is based on instincts alone. At the first step a classification of ideas to interesting and less interesting ones is introduced and less interesting ideas are deleted. At the next level a censorship of dangerous ideas is introduced and the conflict between interesting and dangerous leads to neurotic behaviors, fix idees, and hysteria. As was pointed out by one of referees of this paper, these aspects of the model reflect more the general structure of conscious/unconscious processing rather than properties of $m$-adic numbers. The basic mathematical structure for this model is mental ultrametric space. In particular, ultrametric is used to classify ideas - to assign to each idea its measures of interest and interdiction.

Finally, we apply our approach to mathematical modeling of Freud's theory, see, for example, Freud, 1933, of interaction between unconscious and conscious domains. One of basic features of our model is splitting the process of thinking into two separate (but at the same time closely connected) domains: conscious and unconscious, cf. Freud, 1933. We shall use the following point of view on the simultaneous work of the consciousness and unconsciousness. The consciousness contains a control center $C C$ that has functions of control over results of functioning of subconsciousness. $C C$ formulates problems, and sends them to the unconscious domain. The process of finding a solution is hidden in the unconscious domain. In the unconscious domain there work gigantic dynamical systems - thinking processors. Each processor is determined by a function $f$ from mental space into itself (describing the corresponding feedback process - psychological function). It produces iterations of mental states (points of mental space) $x_{1}=f\left(x_{0}\right), \ldots, x_{n+1}=f\left(x_{n}\right), \ldots$ These intermediate mental states are not used by the consciousness. The consciousness (namely $C C$ ) controls only some exceptional moments in the work of the dynamical system in the unconscious domain - attractors and cycles. Dynamics of mental points induce dynamics of mental figures, in particular, ball-associations and, hence, ideas (collections of balls). The crucial point is that behaviors of the dynamical in the mental space and its lifting to spaces of associations and ideas can be very different. Extremely cycling (chaotic) behavior on the level of mental

\footnotetext{
${ }^{1}$ We do not try to discuss general philosophical and cognitive problems of modeling of consciousness, see, e.g., Blomberg et al. 1994, Baars, 1997, Pitkänen, 1998, Khrennikov, 1998a, 2004a.
} 
states can imply nice stabilization to attractors on the level of ideas.

To couple our hierarchic $m$-adic model of processing of information in brain with other investigations in cognitive brain research, we can mention e.g. the paper of Oztop et al, 2005, presenting an approach to "mental state inference" (oriented toward visual feedback control). We remark that "mental state inference", more generally "theory of mind" has been a resent topic of interest in cognitive neuroscience, see Blakemore and Decety, 2001, Chaminade et al., 2001, Frith C.D. and Frith U., 1999. In fact, we present a model of mental state inference serving for Freud's psychoanalysis. Our model is about estimation of mental states on the basis of symptoms (in the mentioned papers mental states were estimated on the behavioral basis).

Geometrically we can imagine a system of $m$-adic integers (which will be the mathematical basis of our cognitive models) as a homogeneous tree with $m$-branches splitting at each vertex. The distance between mental states is determined by the length of their common root: close mental states have a long common root. The corresponding geometry strongly differs from the ordinary Euclidean geometry.

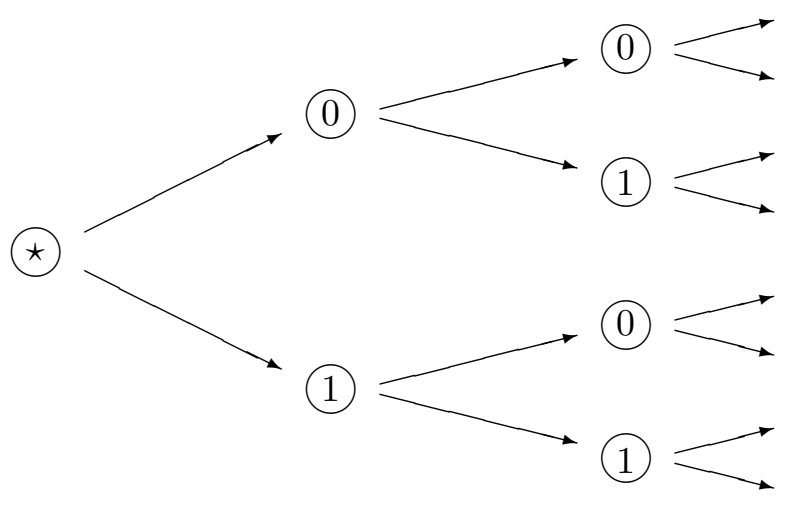

Figure 1: The 2-adic tree

We also point out that systems of $m$-adic numbers (restricted to $m=p$ a prime number) were intensively used in theoretical physics, see e.g. Khrennikov, 1997.

We remark that in this paper we do not consider in details the neuronal basis of the $m$-adic mental space, see Khrennikov 2004a, b, for corresponding models, see section 5 for a brief review. This neuronal basis is provided by consideration of hierarchical neuronal trees. Such trees provides connection of the mental space (produced by a tree) with physical space (in that neuronal trees are located). Mental processes are connected with physical and chemical processes in the brain: mental states are produced as distributed 
activations of neuronal pathways. We remark that mental hierarchy was discussed a lot, see already mentioned papers Smythies, 1970, Clark, 1980, Amit, 1989, Hubel and Wiesel, 1962, Bechtel and Abrahamsen, 1991, Ivanitsky, 1999, Watt and Phillips, 2000, Stringer and Rolls, 2002, Khrennikov, 1997, 1998a,b, 1999a,b, 2000a, b; Albeverio et al., 1999; Dubischar et al, 1999, Voronkov, 2002a, b, Sergin, 2007. However, there were not so much experimental neurophysiological evidences of existence of such neuronal hierarchical structures in the brain. Therefore the recent paper of Luczak et al., 2007, that confirmed experimentally existence of neurons-directors which rule the performances of cognitive tasks (under the same context and learning conditions) is extremely important for our model. The presence of a complicated hierarchy of time scales in the brain can be considered as an indirect confirmation of the hierarchical structure of processing of information in the brain, see, e.g., Geissler et al (1978), Geissler and Puffe (1982), Geissler (1983, 85, 87,92), Geissler and Kompass (1999, 2001), Geissler, Schebera, and Kompass (1999), Klix and van der Meer (1978), Kristofferson (1972, 80, 90), Bredenkamp (1993), Teghtsoonian (1971).

We start with recollection of the basic notions from e.g. Khrennikov, 1997, 1998a,b, 1999a,b, 2000a,b; Albeverio et al., 1999; Dubischar et al, 1999. Consequences for neurophysiology, neuroinformatics, and cognitive sciences as well as for psychology and neuropsychology, and even medicine and psychiatry will be presented in sections 11-13; possibilities to apply our model for the project on artificial life will be considered in section 14 ("psychological robots").

\section{$2 m$-adic ultrametric spaces}

The notion of a metric space is used in many applications for describing distances between objects. Let $X$ be a set. A function $\rho: X \times X \rightarrow \mathbf{R}_{+}$(where $\mathbf{R}_{+}$is the set of positive real numbers) is said to be a metric if it has the following properties: 1) $\rho(x, y)=0$ iff $x=y$ (non-degenerated); 2$) \rho(x, y)=$ $\rho(y, x)$ (symmetric); 3$) \rho(x, y) \leq \rho(x, z)+\rho(z, y) \quad$ (the triangle inequality). The pair $(X, \rho)$ is called a metric space.

We are interested in the following class of metric spaces $(X, \rho)$. Every point $x$ has the infinite number of coordinates

$$
x=\left(\alpha_{1}, \ldots, \alpha_{n}, \ldots\right) .
$$

Each coordinate yields the finite number of values,

$$
\alpha \in A_{m}=\{0, \ldots, m-1\},
$$

where $m>1$ is a natural number, the base of the alphabet $A_{m}$. The metric $\rho$ should be so called ultrametric, i.e., satisfy the strong triangle inequality:

$$
\rho(x, y) \leq \max [\rho(x, z), \rho(z, y)], x, y, z \in X \text {. }
$$


The strong triangle inequality can be stated geometrically: each side of a triangle is at most as long as the longest one of the two other sides. Such a triangle is quite restricted when considered in the ordinary Euclidean space.

We denote the space of sequences (2), (3) by the symbol $\mathbf{Z}_{m}$. The standard ultrametric is introduced on this set in the following way. For two points $x=\left(\alpha_{0}, \alpha_{1}, \alpha_{2}, \ldots, \alpha_{n}, \ldots ..\right), y=\left(\beta_{0}, \beta_{1}, \beta_{2}, \ldots, \beta_{n}, \ldots\right) \in \mathbf{Z}_{m}$,

we set

$$
\rho_{m}(x, y)=\frac{1}{m^{k}} \text { if } \alpha_{j}=\beta_{j}, j=0,1, \ldots, k-1, \text { and } \alpha_{k} \neq \beta_{k} .
$$

This is a metric and even an ultrametric. To find the distance $\rho_{m}(x, y)$ between two strings of digits $x$ and $y$ we have to find the first position $k$ such that strings have different digits at this position.

Let $(X, \rho)$ be an arbitrary ultrametric space. For $r \in \mathbf{R}_{+}, a \in X$, we set

$$
U_{r}(a)=\{x \in X: \rho(x, a) \leq r\}, U_{r}^{-}(a)=\{x \in X: \rho(x, a)<r\} .
$$

These are balls of radius $r$ with center $a$. Balls have the following properties, Khrennikov, 1997:

1) Let $U$ and $V$ be two balls in $X$. Then there are only two possibilities: (a) balls are ordered by inclusion (i.e., $U \subset V$ or $V \subset U$ ); (b) balls are disjoint 2

2) Each point of a ball may serve as a centre.

3) In some ultrametric spaces a ball may have infinitely many radii.

Let $m>1$ be the fixed natural number. We consider the $m$-adic metric space $\left(\mathbf{Z}_{m}, \rho_{m}\right)$. This metric space has the natural algebraic structure, see Khrennikov, 1997.

A point $x=\left(\alpha_{0}, \alpha_{1}, \alpha_{2}, \ldots, \alpha_{n}, \ldots\right)$ of the space $\mathbf{Z}_{m}$ can be identified with a so called $m$-adic number:

$$
x=\alpha_{0} \alpha_{1} \ldots \alpha_{k} \ldots \equiv \alpha_{0}+\alpha_{1} m+\ldots+\alpha_{k} m^{k}+\ldots .
$$

The series (5) converges in the metric space $\mathbf{Z}_{m}$. In particular, a finite mental string $x=\alpha_{0} \alpha_{1} \ldots \alpha_{k}$ can be identified with the natural number

$$
x=\alpha_{0}+\alpha_{1} m+\ldots+\alpha_{k} m^{k} .
$$

Therefore the set of all finite mental strings can be identified with the set of natural numbers $\mathbf{N}$. So dynamics of finite mental strings can be simulated via dynamics on $\mathbf{N}$. Moreover, $\mathbf{N}$ is a dense subset of $\mathbf{Z}_{m}$ : each $x \in \mathbf{Z}_{m}$ can be approximated with an arbitrary precision by natural numbers. Thus the space $m$-adic numbers can be considered as an extension of the set of natural numbers. By choosing different $m$ we obtain in general different extensions $\mathbf{Z}_{m}$. Therefore we can say that in our model mental states are

\footnotetext{
${ }^{2}$ There is the third possibility in the Euclidean space .
} 
encoded by natural numbers. However, the mental geometry on the set of natural numbers differs crucially from the one which is induced from the real line.

It is possible to introduce algebraic operations on the set of $m$-adic numbers $\mathbf{Z}_{m}$, namely addition, subtraction, and multiplication. These operations are natural extensions by the $m$-adic continuity of the standard operations on the set of natural numbers $\mathbf{N}=\{0,1,2,3, \ldots\}$.

\section{Mental Space}

We shall use the following mathematical model for mental space:

(1) Set-structure: The set of mental states $X_{\text {mental }}$ has the structure of the $m$-adic tree: $X_{\text {mental }}=\mathbf{Z}_{m}$.

(2) Topology: Two mental states $x$ and $y$ are close if they have sufficiently long common root. This topology is described by the metric $\rho_{m}$.

In our mathematical model mental space is represented as the metric space $\left(\mathbf{Z}_{m}, \rho_{m}\right)$.

Dynamical thinking on the level of mental states is performed via the following procedure: an initial mental state $x_{0}$ is sent to the unconscious domain; it is iterated by some dynamical system which is determined by a map

$$
f: \mathbf{Z}_{m} \rightarrow \mathbf{Z}_{m}
$$

an attractor is communicated to the consciousness; this is the solution of a problem $x_{0}$ 3 Our mathematical model is based on two cornerstones:

$\mathrm{H})$. The first is the assumption that the coding system which is used by the brain for recording vectors of information generates a hierarchical structure between digits of these vectors. Thus if $x=\left(\alpha_{1}, \alpha_{2}, \ldots, \alpha_{n}, \ldots\right), \alpha_{j}=$ $0,1, \ldots, m-1$, is an information vector which presents in the brain a mental state then digits $\alpha_{j}$ have different weights. The digit $\alpha_{0}$ is the most important, $\alpha_{1}$ dominates over $\alpha_{2}, \ldots, \alpha_{n}, \ldots$, and so on.

D). The second is the assumption that functioning of the brain is not based on the rule of reason. The unconsciousness is a collection of dynamical systems $f_{s}(x)$ (thinking processors) which produce new mental states practically automatically. The consciousness only uses and control results (attractors in spaces of ideas) of functioning of unconscious processors.

\footnotetext{
${ }^{3}$ It may be that iterations starting with some $x_{0}$ will not arrive to any attractor. For example, starting with $x_{0}, \tau$ may perform a cyclic behavior in the process of thinking. In such case a cognitive system $\tau$ would not find the definite solution of a problem. In particular, it is impossible to escape a cyclic behavior on the level of mental states: even the simplest dynamical systems in $\mathbf{Z}_{m}$ may have a huge number of cycles, see Khrennikov, 1997.
} 
For a neuronal basis of the $m$-adic mental space, see Khrennikov 2004a, b. We also mention the possibility to apply the hierarchic mental space to genetics.

Example 3.1 (4-adic genetic information space) We may describe DNA and RNA sequences by $\mathrm{m}$-adic numbers. We present schematically development of this model. DNA and RNA sequences are represented by 4 -adic numbers. Nucleotides are mapped to digits in registers of 4-adic numbers: adenine - $A$, guanine - $G$, cytosine - $C$, and thymine - $T$ are encoded by $\alpha=0,1,2,3$. The $U$-nucleotide is represented by 3. The DNA and RNA sequences have the natural hierarchic structure: letters which are located at the beginning of a chain are considered as more important. This hierarchic structure coincides with the hierarchic structure of the 4-adic tree. It can also be encoded by the 4-adic metric. The process of DNA-reproduction is described by action of 4-adic dynamical system. As we know, the genes contain information for production of proteins. The genetic code is a degenerate map of codons to proteins. We model this map as functioning of a monomial 4-adic dynamical system. Proteins are attractors of this dynamical system. We also can study the process the genom evolution in the framework of 4-adic dynamical systems.

\section{Associations and Ideas}

We now improve this dynamical cognitive model on hierarchic mental trees by introducing a new hierarchy: equivalence classes of mental states are interpreted as associations, collections of associations as ideas.

A new property of dynamics of ideas is that (for a large class of dynamical systems on $m$-adic trees) for each initial idea $J_{0}$ its iterations are attracted by some idea $J_{\text {attr }}$, see Khrennikov, 1997, 2004a, for mathematical details. The latter idea is considered by the consciousness as a solution of the problem $J_{0}$. In the opposition to such an attractor-like dynamics of ideas, dynamics of mental states (on the tree $X_{\text {mental }}$ ) or associations need not be attractive. In particular, there can exist numerous cycles or ergodic evolution.

By using higher cognitive levels (associations and ideas) of the representation of information a cognitive system strongly improves the regularity of thinking dynamics. Finally, we note that the use of a new cognitive hierarchy (in combination with the basic hierarchy of the $m$-adic tree) strongly improves the information power of a cognitive system.

Special collections of mental points form new cognitive objects, associations. Let $s \in\{0,1, \ldots, m-1\}$. A set

$$
A_{s}=\left\{x=\left(\alpha_{0}, \ldots, \alpha_{k}, \ldots\right) \in \mathbf{Z}_{m}: \alpha_{0}=s\right\}
$$

is called an association of order 1. By realizing $\mathbf{Z}_{m}$ as the metric space we see that $A_{s}$ can be represented as the ball of radius $r=\frac{1}{m}$. Any point $a$ having $\alpha_{0}$ as the first digit can be chosen as a center of this ball (we recall that in an 
ultrametric space any point $a$ belonging to a ball can be chosen as its center): $A_{s}=U_{\frac{1}{m}}(a)$, where $a=\left(a_{0}, a_{1}, \ldots\right), a_{0}=s$. Associations of higher orders are defined in the same way. Let $s_{0}, \ldots, s_{k-1} \in\{0,1, \ldots, m-1\}$. The set

$$
A_{s_{0} \ldots s_{k}}=\left\{x=\left(\alpha_{0}, \ldots, \alpha_{k}, . .\right) \in \mathbf{Z}_{m}: \alpha_{0}=s_{0}, \ldots, \alpha_{k-1}=s_{k-1}\right\}
$$

is called an association of order $k$. These are balls of the radius $r=\frac{1}{m^{k}}$.

Denote the set of all associations by the symbol $X_{A}$. Collections of associations will be called ideas. Denote the set of all ideas by the symbol $X_{I D}$. The space $X_{I D}$ consists of points-associations.

In this section we study the simplest dynamics of associations and ideas which are induced by corresponding dynamics of mental states,

$$
x_{n+1}=f\left(x_{n}\right) .
$$

Suppose that, for each association, its image is again an association. Thus $f$ maps balls onto balls. Then dynamics (6) of mental states of $\tau$ induces dynamics of associations

$$
A_{n+1}=f\left(A_{n}\right) .
$$

We say that dynamics in the mental space $X_{\text {mental }}$ for transformations is lifted to the space of associations $X_{A}$.

\section{Neuronal Realization}

Let us consider the simplest model of a neuronal tree $T_{\text {neuronal }}$ inducing a mental space $X_{\text {mental }}$. This model is based on the 2-adic neuronal tree given by Figure 1. Each vertex of this tree corresponds to a single neuron. In this idealized model each axon provides connections with precisely two neurons of lower level of the hierarchy in the neuronal tree. There is the root-neuron denoted by $\star$, its axon provides connections with the two neurons, 0 and 1 , of the lower level. Each of these neurons sends its axon to precisely two neurons of the lower level and so on. Each branch $n$ of this tree ( a hierarchical chain of neurons) can be coded by a sequence of zeros/ones Thus this neuronal tree can be mathematically represented as the set of 2 -adic numbers: $T_{\text {neuronal }}=$ $\mathbf{Z}_{2}$.

Each branch of this neuronal tree is a device for producing mental states (cognitive mental images). In the simplest model we suppose that each neuron can be only in the two states: $\alpha=1$, firing, $\alpha=0$, non-firing. Thus each branch produces (at some moment of time) a sequence of zeros/ones: $x=\alpha_{0} \alpha_{1} \ldots \alpha_{N} \ldots$, where $\alpha_{j}=0,1$. (In the mathematical model we can consider infinitely long sequences). Thus the neuronal tree $T_{\text {neuronal }}=\mathbf{Z}_{2}$ produces the mental space $X_{\text {mental }}=\mathbf{Z}_{2}$. We can consider a mental field on the neuronal tree $T_{\text {neuronal }}$. This is the map

$$
\psi: T_{\text {neuronal }} \rightarrow X_{\text {mental }},
$$


mathematically:

$$
\psi: \mathbf{Z}_{2} \rightarrow \mathbf{Z}_{2}, \psi(n)=x .
$$

In fact, we need not assume that the 2-adic mental space should be based on the 2-adic morphology of the neuronal tree. In general there is no direct connection between the morphology of a the neuronal tree and the corresponding mental space. Let us consider any tree $T_{\text {neuronal }}$ with the root $\star$ (any number of edges leaving a vertex, so an axon can provide connections with any number of neurons at the lower floor). Nevertheless, let us consider the same firing/not coding system. Each branch of the neuronal tree $T_{\text {neuronal }}$ produces a 2-adic number. Here the mental field is a $\mathbf{Z}_{2}$-valued function on $T_{\text {neuronal }}$. This is an important property of the model: it would be not so natural to consider only homogeneous neuronal trees of the $m$-adic type.

The structure of the mental space is determined not by the morphology of the neuronal tree, but by the coding system for states of neurons.

Let us consider more advanced system of coding based on frequencies of spiking for neurons, e.g., Hoppensteadt, 1997. We assign to each neuron its frequency of spiking:

$$
\alpha=k, \text { for the frequency } \nu=\frac{2 \pi k}{m}, k=0,1, \ldots, m-1 .
$$

Such a system of coding induces the $m$-adic mental space, $X_{\text {mental }}=\mathbf{Z}_{m}$ for any neuronal tree. Each mental function is based on its own neuronal tree: $T_{\text {neuronal }}=T_{\text {neuronal }}(f)$.

\section{Model of Cognitive Psychology}

We point out that the model which is developed in this paper is a model of neuropsychology and not at all a model of neurophysiology. The neuronal trees under consideration are not trees for integration-propagation of sensory stimuli forming new mental categories at each level of such a tree (see Khrennikov, 2002, for a general model). We consider neuronal trees creating associations. As an example, let us consider a neuronal tree which is used for representation of persons. There can be used various hierarchical representations. We choose the "sex-representation": the state of the root-neuron, $\star$, gives the sex of a person: $\alpha_{0}=1-$ female, $\alpha_{0}=0-$ male. Consider a branch of this tree. Suppose that in this representation the next neuron (after $\star)$ gives the age of a person: $\alpha_{1}=1-$ young, $\alpha_{1}=0-$ not, and so on: $\alpha_{2}=1-$ blond, $\alpha_{2}=0-$ not, $\alpha_{3}=1-$ high education $/$ not, $\ldots$

Take the ball $U_{1 / 2}=\left\{x=\alpha_{0} \alpha_{1} \ldots \alpha_{N} \ldots: \alpha_{0}=1\right\}$. This is the association of woman.

Take the ball $U_{1 / 4}=\left\{x=\alpha_{0} \alpha_{1} \ldots \alpha_{N} \ldots: \alpha_{0}=1, \alpha_{1}=1\right\}$. This is the association of young woman.

Take the ball $U_{1 / 8}=\left\{x=\alpha_{0} \alpha_{1} \ldots \alpha_{N} \ldots: \alpha_{0}=1, \alpha_{1}=1, \alpha_{2}=1\right\}$. This is the association of young blond woman. 
Take the ball $U_{1 / 16}=\left\{x=\alpha_{0} \alpha_{1} \ldots \alpha_{N} \ldots: \alpha_{0}=1, \alpha_{1}=1, \alpha_{2}=\right.$ $\left.1, \alpha_{3}=1\right\}$. This is the association of young blond woman with high education.

Take now the ball $V_{1 / 4}=\left\{x=\alpha_{0} \alpha_{1} \ldots \alpha_{N} \ldots: \alpha_{0}=0, \alpha_{1}=1\right\}$. This is the association of young man.

Take the union of two balls-associations: $W=U_{1 / 4} \cup V_{1 / 4}=\{x=$ $\left.\alpha_{0} \alpha_{1} \ldots \alpha_{N} \ldots: \alpha_{1}=1\right\}$. This is an idea of young person.

"Young person", $W$, is an idea with respect to the hierarchy based on sex. If we consider another hierarchy (based on another neuronal tree) for that the root-neuron represents not sex, but age, then it produces "young person" as the association: $U_{1 / 2}^{\text {age }}=\left\{y=\beta_{0} \beta_{1} \ldots \beta_{N} \ldots: \beta_{0}=1\right\}$.

But $U_{1 / 2}^{\text {age }}$ is not completely the same mental object as $W$. The $U_{1 / 2}^{\text {age }}$ is the "unisex young person" and $W$ "young person with sex."

We see that our considerations of changing of neuronal trees and hence mental representations is similar to the choice of coordinate systems in physics.

As was remarked at the beginning of this section, dynamics of associations and ideas need not be based on external stimuli (sensor or mental). Thus a neuronal tree can be self-activated even without signals from outside, cf. with experimental results Luczak et al., 2007.

\section{Dynamics of Associations and Ideas}

Dynamics of associations (7) automatically induces dynamics of ideas

$$
J_{n+1}=f\left(J_{n}\right) .
$$

Geometrically associations are represented as bundles of branches of the $m$ adic tree. Ideas are represented as sets of bundles. Thus dynamics (6), (7), (9) are, respectively, dynamics of branches, bundles and sets of bundles on the $m$-adic tree. To give examples of $f$ mapping balls onto balls, we use the standard algebraic structure on $\mathbf{Z}_{m}$. For example, it is known, Khrennikov 1997, 2004a, that all monomial dynamical systems belong to this class.

We are interested in attractors of dynamical system (9) (these are ideassolutions). To define attractors in the space of ideas $X_{I D}$, we have to define a convergence in this space. We must introduce a distance on the space of ideas (sets of associations). Unfortunately there is a small mathematical complication. A metric on the space of points does not induce a metric on the space of sets that provides an adequate description of the convergence of ideas. It is more useful to introduce a generalization of metric, namely so called pseudometric. 4 Hence dynamics of ideas is a dynamics not in a metric space, but in more general space, so called pseudometric space.

\footnotetext{
${ }^{4}$ In fact, it is possible to introduce even a metric (Hausdorff's metric) as people in general topology do. However, it seems that this metric does not give an adequate description of dynamics of associations and ideas.
} 
Let $(X, \rho)$ be a metric space. The distance between a point $a \in X$ and a subset $B$ of $X$ is defined as

$$
\rho(a, B)=\inf _{b \in B} \rho(a, b)
$$

(if $B$ is a finite set, then $\rho(a, B)=\min _{b \in B} \rho(a, b)$ ).

Denote by $\operatorname{Sub}(X)$ the system of all subsets of $X$. Hausdorff's distance between two sets $A$ and $B$ belonging to $\operatorname{Sub}(X)$ is defined as

$$
\rho(A, B)=\sup _{a \in A} \rho(a, B)=\sup _{a \in A} \inf _{b \in B} \rho(a, b) .
$$

If $A$ and $B$ are finite sets, then

$$
\rho(A, B)=\max _{a \in A} \rho(a, B)=\max _{a \in A} \min _{b \in B} \rho(a, b) .
$$

Hausdorff's distance $\rho$ is not a metric on the set $Y=\operatorname{Sub}(X)$. In particular, $\rho(A, B)=0$ does not imply that $A=B$. Nevertheless, the triangle inequality $\rho(A, B) \leq \rho(A, C)+\rho(C, B), \quad A, B, C \in Y$, holds true for Hausdorff's distance.

Let $T$ be a set. A function $\rho: T \times T \rightarrow \mathbf{R}_{+}=[0,+\infty)$ for which the triangle inequality holds true is called a pseudometric on $T ;(T, \rho)$ is called a pseudometric space. Hausdorff's distance is a pseudometric on the space $Y$ of all subsets of the metric space $X ;(Y, \rho)$ is a pseudometric space. The strong triangle inequality $\rho(A, B) \leq \max [\rho(A, C), \rho(C, B)] \quad A, B, C \in Y$, holds true for Hausdorff's distance corresponding to an ultrametric $\rho$ on $X$. In this case Hausdorff's distance $\rho$ is an ultra-pseudometric on the set $Y=$ $\operatorname{Sub}(\mathrm{X})$.

\section{Advantages of dynamical processing of associ- ations and ideas}

As was already mentioned, the main distinguishing feature of the dynamics of associations and ideas are their regularity comparing with the dynamics of mental states. Typically the dynamics of mental states is irregular. Numerous cycles and ergodic components appear and disappear depending on $m$. Moreover, dynamics on more complex mental spaces (larger $m$ and more floors for mental trees) is more irregular than dynamics on simpler mental spaces. Thus cognitive systems having more complex brains would have real problems with successive processing of information, i.e., with obtaining attractors-solutions (of course, under the assumption that our model for the hierarchical dynamical processing of mental information is adequate to the functioning of the real brain).

Surprisingly such an irregularity for mental states induces the regular dynamics of associations and ideas, Khrennikov 1997, 2004a. Cycles of states 
disappear. They are hidden in balls-associations. Ergodic components are also unified into balls-associations.

Thus by using the associative representation of mental information the brain working as a collection of dynamical systems on hierarchical trees essentially increases the regularity of information processing. By our model primitive brains (having a few levels of mental hierarchy and rather weak networks of connections between hierarchical levels) are fine by working only with mental states. However, a more complex brains should form associations to stabilize dynamical processing of information.

\section{Transformation of unconscious mental flows into conscious flows}

We represent a few mathematical models of the information architecture of conscious systems $\tau$, cf., e.g., Fodor and Pylyshyn, 1988, Edelman, 1989, Voronkov, 2002a. We start with a quite simple model (Model 1). This model will be developed to more complex models which describe some essential features of human cognitive behavior. The following sequence of cognitive models is related to the process of evolution of the mental architecture of cognitive systems.

\subsection{Model 1}

A). The brain of $\tau$ is split into two domains: conscious and unconscious.

B). There are two control centers, namely a conscious control center $C C$ and an unconscious control center $U C$.

C). The main part of the unconscious domain is a processing domain $\Pi$. Dynamical thinking processors $\pi$ are located in $\Pi$.

In the simplest case the outputs of some group of thinking processors $\pi_{\mathrm{un}}^{1}, \ldots, \pi_{\mathrm{un}}^{n}$ are always sent to $U C$ and the outputs of another group $\pi_{\mathrm{c}}^{1}, \ldots, \pi_{\mathrm{c}}^{m}$ are always sent to $C C 5$ The brain of $\tau$ works in the following way.

External information is transformed by $C C$ into some problem-idea $J_{0}$. The $C C$ sends $J_{0}$ to a thinking processor $\pi$ located in the domain $\Pi$. Starting with $J_{0}, \pi$ produces via iteration $J_{1}, \ldots, J_{N}, \ldots$ an idea-attractor $J$.

If $\pi=\pi_{\mathrm{un}}^{i}$ (one of the unconscious-output processors), then $J$ is transmitted to the control center $U C$. This center sends $J$ as an initial idea $J_{0}^{\prime}=J$ to $\Pi$ or to a physical (unconscious) performance. In the first case some $\pi^{\prime}$ (it can be conscious - output as well as unconscious - output processor) performs iterations $J_{0}^{\prime}, J_{1}^{\prime}, \ldots, J_{N}^{\prime}, \ldots$ and produces a new idea-attractor $J^{\prime}$. In the second case $J$ is used as a signal to some physiological system.

\footnotetext{
${ }^{5}$ Information produced by $\pi_{\text {un }}$ cannot be directly used in the conscious domain. This information circulates in the unconscious domain. Information produced by $\pi_{\mathrm{c}}$ can be directly used in the conscious domain.
} 
If $\pi=\pi_{c}^{i}$ (one of conscious-output processors), then $J$ is transmitted to the control center $C C$. This center sends $J$ as an initial idea $J_{0}^{\prime}=J$ to $\Pi$ (to some $\pi^{\prime}$ ) or to a physical or mental performance (speech, writing), or to memory. There is no additional analysis of an idea-attractor $J$ which is produced in the unconscious domain. Each attractor is recognized by the control center $C C$ as a solution of the initial problem $J_{0}$, compared with models 2-4.

Moreover, it is natural to assume that some group of thinking processors $\pi_{\Pi}^{1}, \ldots, \pi_{\Pi}^{l}$ have their output only inside the processing domain $\Pi$. An attractor $J$ produced by $\pi_{\Pi}$ is transmitted neither to $C C$ nor to $U C$. The $J$ is directly used as the initial condition by some processor $\pi$. Finally, we obtain the mental architecture of a brain given by Figure 2 .

In this model $C C$ sends all ideas obtained from the unconscious domain to realization: mental or physical performance, memory recording, transmission to $\Pi$ for a new cycle of the process of thinking. If the intensity of the flow of information from the unconscious domain is rather high, then such a $\tau$ can have problems with realizations of some ideas.

Example 9.1. (Primitive love). Let $\tau$ be a 'man' described by this model and let $\pi=\pi_{\text {sex }}$ be his sexual thinking system. The image $J_{0}$ of a woman $\gamma$ is sent by $C C$ to $\pi_{\text {sex }}$. This thinking block performs iterations $J_{0}, J_{1}, \ldots, J_{N}$ and produces an idea - attractor $J$. In the simplest case we have the pathway: $C C \rightarrow \pi_{\text {sex }} \rightarrow C C$ (in principle, there could be extremely complex and long pathways, for example, $C C \rightarrow \pi_{\text {sex }} \rightarrow \pi^{\prime} \rightarrow \pi^{\prime \prime} \rightarrow U C \rightarrow \pi^{\prime \prime \prime} \rightarrow C C$ ). Suppose that the idea $J_{\text {love }}=($ love $\gamma$ ) is the attractor for iterations starting with the image $J_{0}$ of a woman. Then $J_{\text {love }}$ is sent directly to realization. Thus $\tau$ has no doubts and even no craving. He performs all orders of the unconscious domain. In fact, $C C$ can be considered as a simple control device performing the connection with the external world. The $\tau$ could not have mental problems. The only problem for $\tau$ is an intensive flow of images of women. This problem can be solved if $\tau$ collects images and then chooses randomly an image for realization.

The reader may ask: Why does such a cognitive system $\tau$ need to split mental processing into conscious and unconscious domains? The main consequence of this splitting is that the $\tau$ does not observe iterations of dynamical systems performing intensive computations. The consciousness, CC, pays attention only to results (attractors) of functioning of thinking processors. As a consequence, the $\tau$ is not permanently disturbed by these iterations. It can be concentrated on processing of external information and final results of the process of thinking.

Besides the unconscious control center $U C$ and the processing domain $\Pi$, the unconscious domain contains some other structures (empty boxes of this picture). These additional structures (in the conscious as well as unconscious domains) will be introduced in more complex models. We shall also describe the character of connections between $C C$ and $U C$. In general we need 


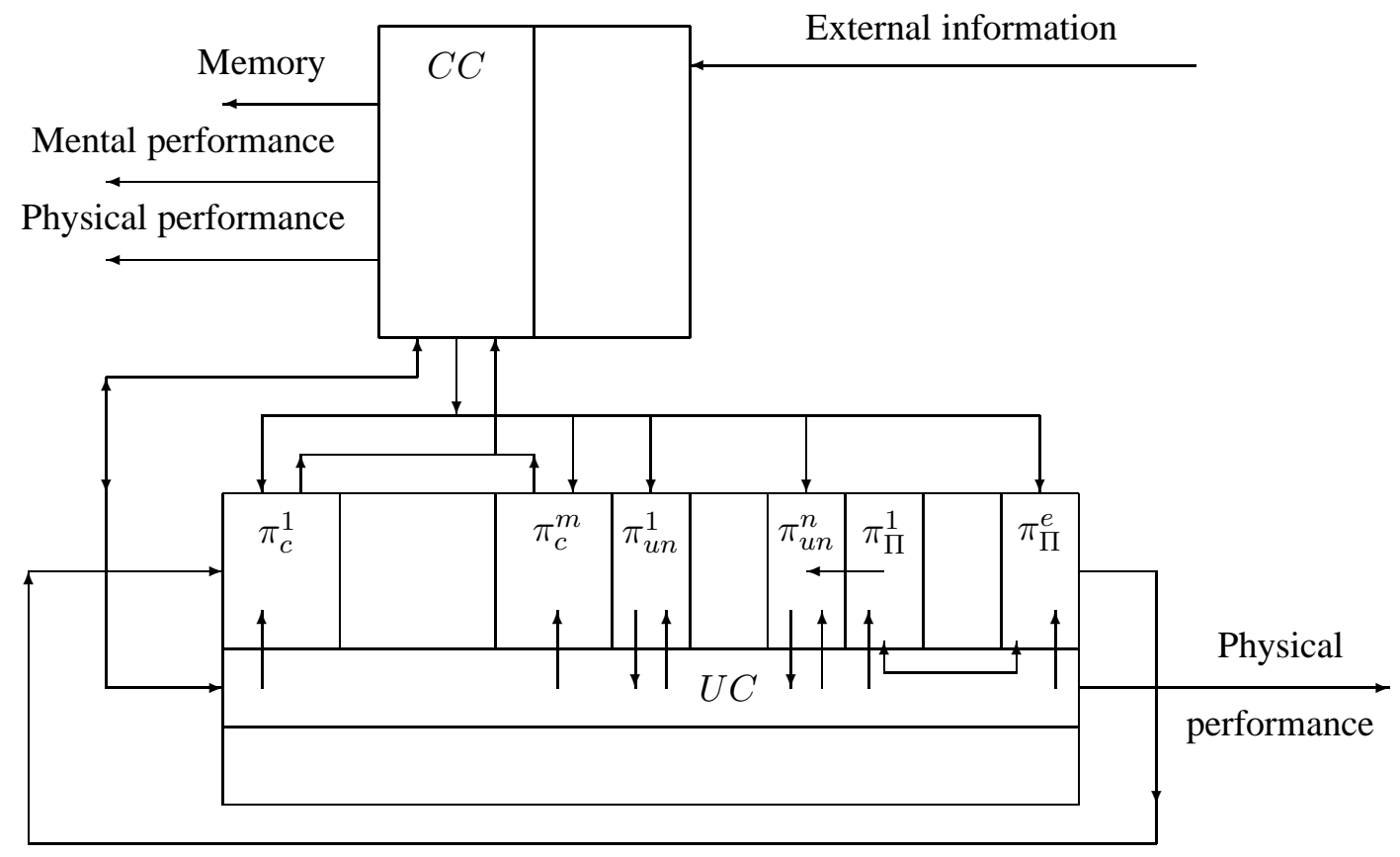

Unconscious domain

Figure 2: Model 1 of conscious/unconscious functioning 
not assume the specialization of processors $\pi$ in $\Pi:\left(\pi_{\mathrm{c}}^{1}, \ldots, \pi_{\mathrm{c}}^{m}\right) \rightarrow C C$, $\left(\pi_{\mathrm{un}}^{1}, \ldots, \pi_{\mathrm{un}}^{n}\right) \rightarrow U C,\left(\pi_{\Pi}^{1}, \ldots, \pi_{\Pi}^{l}\right) \rightarrow\left(\pi_{\Pi}^{1}, \ldots, \pi_{\Pi}^{l}\right)$.

\subsection{Model 2}

One of the possibilities to improve functioning of $\tau$ is to create a queue of ideas $J$ waiting for a realization. Thus it is natural to assume that the conscious domain contains some collector $Q$ in that all 'waiting ideas' are gathered.

Ideas in $Q$ must be ordered for successive realizations. The same order structure can be used to delete some ideas if $Q$ is complete. Thus all conscious ideas must be classified.

They obtain some characteristics $I(J)$ that gives a measure of interest to an idea $J$. We may assume that $I$ takes values in some segment $[\delta, 1]$ (in the $m$-adic model $\delta=1 / 2$, see Remark 9.1). If $I(J)=1$, then an idea $J$ is extremely interesting for $\tau$. If $I(J)=\delta$, then $\tau$ is not at all interested in $J$. There exists a threshold $I_{\mathrm{rz}}$ of the minimal interest for realization. If $I(J)<I_{\mathrm{rz}}$, then the control center $C C$ directly deletes $J$, despite the fact that $J$ was produced in the unconscious domain as the solution of some problem $J_{0}$. If $I(J) \geq I_{\mathrm{rz}}$, then $C C$ sends the idea $J$ to $Q$.

The $\tau$ lives in the continuously changed environment. The $\tau$ could not be concentrated on realization of only old ideas $J$ even if they are interesting. Realizations of new ideas which are related to the present instant of time $t$ can be more important. The time-factor must be taken into account.

Let $l(t), l(0)=1$, be some function (depending on $\tau$ ) which decreases with the increasing of time $t$. Suppose that the interest $I(t, J)$ of an idea $J$ in the queue $Q$ evolves as

$$
I(t, J)=l\left(t-t_{0}\right) I(J),
$$

where $I(J)$ is the value of interest of $J$ at the instant $t_{0}$ of the arrival to the collector $Q$. Thus the interest to $J$ is continuously decreasing. Finally, if $I(t, J)$ becomes less than the realization threshold $I_{\mathrm{rz}}$ the $J$ is deleted from $Q$.

Quick reactions to new circumstances can be based on an exponentially decreasing coefficient $l(t): l(t)=e^{-C t}$, where a constant $C>0$ depends on $\tau$ If an idea $J$ has an extremely high value of interest $I(J) \geq I_{+}$(where $I_{+}$is a preserving threshold), then it must be realized in any case. In our model we postulate that if $I(J) \geq I_{+}$, the interest to $J$ is not changed with time: $I(t, J)=I(J)$. 7 We note that, of course, $I_{+} \geq I_{\mathrm{rz}}$.

\footnotetext{
${ }^{6}$ It may be that the level of interest of $J$ evolves in a more complex way. For example, $I(t, J)=$ $\exp \{-C(J) t\} I(J)$. Here different ideas $J$ have different coefficients $C(J)$ of decreasing interest.

${ }^{7}$ For example $I(t, J)=\exp \{-C(J) t\} I(J)$, where $C(J)=0$ for $I(J) \geq I_{+}$. So $C(J)=$ $\alpha \theta\left(I_{+}-I(J)\right)$, where $\alpha>0$ is a constant (parameter of the brain) and $\theta$ is a Heavyside function: $\theta(t)=1, t \geq 0$, and $\theta(t)=0, t<0$.
} 
We now describe one of the possible models for finding the value $I(J)$ of interest for an idea $J$.

The conscious domain contains a database $D_{i}$ of ideas which are interesting for $\tau$. A part of this database $D_{i}$ was created in the process of evolution. It is transmitted from generation to generation (perhaps even DNA?). A part of the $D_{i}$ is continuously created on the basis of $\tau$ 's experience.

The conscious domain contains a special block, comparator, $\mathrm{COM}_{\mathrm{c}}$ that measures the distance between two ideas, $\rho\left(J_{1}, J_{2}\right)$, and the distance between an idea $J$ and the set $D_{i}$ of interesting ideas: $\rho\left(J, D_{i}\right)$.

At the present level of development of neurophysiology we cannot specify a mental distance $\rho$. Moreover, such a distance may depend on a cognitive system or class of cognitive systems. The hierarchic structure of the process of thinking gives some reasons to suppose that $\mathrm{COM}_{\mathrm{c}}$ might use the $m$-adic pseudometric $\rho_{m}$ on the space of ideas $X_{I D}$. Thus the reader may assume that everywhere below $\rho$ is generated by the $m$-adic metric. However, all general considerations are presented for an arbitrary metric.

We recall that the distance between a point $b$ and a finite set $A$ is defined as $\rho(b, A)=\min _{a \in A} \rho(b, a)$. If $J$ is close to some interesting idea $L_{0} \in D_{i}$, then $\rho\left(J, D_{i}\right)$ is small. In fact, we have $\rho\left(J, D_{i}\right) \leq \rho\left(J, L_{0}\right), L_{0} \in D_{i}$. If $J$ is far from all interesting ideas $L \in D_{i}$, then $\rho\left(J, D_{i}\right)$ is large. We define a measure of interest $I(J)$ as

$$
I(J)=\frac{1}{1+\rho\left(J, D_{i}\right)} .
$$

Thus, $I(J)$ is large if $\rho\left(J, D_{i}\right)$ is small; $I(J)$ is small if $\rho\left(J, D_{i}\right)$ is large.

Remark 9.1. (The range of interest in the $m$-adic model). Suppose that the distance $\rho$ is bounded from above:

$$
\sup _{J_{1}, J_{2}} \rho\left(J_{1}, J_{2}\right) \leq C, \quad J_{1}, J_{2} \in X_{I D}
$$

Then $I(J) \geq \delta=\frac{1}{1+C}$. In such a case ' $I(J)$ is very small' if $I(J) \approx \delta$. The function $I(J)$ takes values in the segment $[\delta, 1]$ (we remark that if $\rho\left(J, D_{i}\right)=$ 0 , then $I(J)=1)$. Let $\rho$ be Hausdorff's pseudometric induced on the space of ideas $X_{I D}$ by the $m$-adic metric $\rho_{m}$. We have $\rho\left(J_{1}, J_{2}\right) \leq 1$ for every pair of ideas $J_{1}, J_{2}$. Here $\delta=1 / 2$ and $I(J)$ always belongs to $[1 / 2,1]$. The sentence ' $I(J)$ is very small' means that $I(J) \approx 1 / 2$ and, as always, ' $I(J)$ is very large' means that $I(J) \approx 1$.

There should be a connection between the level $I(J)$ of interest and the strength of realization of $J$. Signals for mental or physical performances of $J$ increase with increasing of $I(J)$. If, for example, the idea $J=$ to beat this person $\}$ then the strength of the beat increases with increasing of $I(J)$.

In the process of memory recording the value of $I(J)$ also plays an important role. It is natural to suppose that in working memory the evolution of 
the quantity $I(t, J)$ is similar to the evolution which was considered in $Q$ : $I(t, J)=l_{\text {mem }}\left(t-t_{0}\right) I(J)$, where $l_{\text {mem }}(0)=1$ and $l_{\text {mem }}(t)$ decreases with increasing of $t$. If $I(t, J)$ becomes less than the memory preserving threshold $I_{-}^{\mathrm{mem}}$, then $J$ is deleted from working memory.

Example 9.2. (Love with interest). Let $\tau$ be a 'man' described by Model 2. In the same way as in Example 9.1 the image $J_{0}$ of a woman $\gamma$ may produce the idea $J_{\text {love }}=($ love $\gamma)$. However, $J_{\text {love }}$ is not sent to realization automatically. The $\mathrm{COM}_{\mathrm{c}}$ measures $\rho\left(J_{\text {love }}, D_{i}\right)$. Suppose that the database $D_{i}$ of interesting ideas contains the idea (image) $L_{\mathrm{blond}}=$ (blond woman). If $\gamma$ is blond, then $\rho\left(J_{\text {love }}, D_{i}\right)$ is small. So $I\left(J_{\text {love }}\right)$ is large and $C C$ sends $J_{\text {love }}$ to realization. However, if $\gamma$ is not blond, then $J_{\text {love }}$ is deleted (despite the unconscious demand $J_{\text {love }}$ ). Of course, the real situation is more complicated. Each $\tau$ has his canonical image $L_{\text {blond }}$. As $J_{\text {love }}=J_{\text {love; } \gamma}$ depends on $\gamma$, distance $\rho\left(J_{\text {love }}, D_{i}\right)$ can be essentially different for different women $\gamma$. Thus, for one blond woman $\gamma, I\left(J_{\text {love }}\right) \geq I_{\mathrm{rz}}$, but for another blond woman $\gamma, I\left(J_{\text {love }}\right)<I_{\text {rz }}$. If there are few blond women $\gamma_{1}, \ldots, \gamma_{l}$ with $I\left(J_{\text {love } ; \gamma_{\mathrm{j}}}\right) \geq I_{\mathrm{rz}}$, then all ideas $J_{\text {love } \gamma_{\mathrm{j}}}$ are collected in $Q$. The queue of blond women is ordered in $Q$ due to values $I\left(J_{\text {love; } \gamma \mathrm{j}}\right)$. If, for some $\gamma$, $I\left(J_{\text {love } ; \gamma}\right) \geq I_{+}$, then the level of interest toward $J_{\text {love } ; \gamma}$ will not decrease with time. The level of $I\left(J_{\text {love; } \gamma}\right)$ determines the intensity of realization of love with $\gamma$.

The mental architecture of 'brain' in Model 2 is given by Figure 3: A new block $\mathrm{COM}_{\mathrm{c}}$ in the conscious domain measures the distance between an idea-attractor $J$ which has been produced in the unconscious domain and the database $D_{i}$ of interesting ideas. This distance determines the level of interest for $J: I(J)=1 /\left(1+\rho\left(J, D_{i}\right)\right)$. Ideas waiting for realization, $J^{1}, \ldots, J^{s}$, are collected in the special collector $Q$. They are ordered by values of $I(J): I\left(J^{1}\right) \geq I\left(J^{2}\right) \geq \ldots \geq I\left(J^{s}\right) \geq I_{+}$. If $I(J) \geq I_{+}$ (where $I_{+}$is the preserving threshold), then the value of interest of $J$ does not decrease with time.

\subsection{Model 3}

The life of $\tau$ described by Model 2 is free of contradictions. The $\tau$ is always oriented to realizations of the most interesting ideas, wishes, desires. However, environment (and, in particular, social environment) produces some constraints to realizations of some interesting ideas.

In a mathematical model we introduce a new quantity $F(J)$ which describes a measure of interdiction for an idea $J$.

It can be again assumed that $F(J)$ takes values in the segment $[\delta, 1]$. Ideas $J$ with small $F(J)$ have low levels of interdiction. If $F(J) \approx \delta$, then $J$ is a 'free idea'. Ideas $J$ with large $F(J)$ have high levels of interdiction. If $F(J) \approx 1$, then $J$ is totally forbidden. 
The interdiction function is computed in the same way as the interest function. The conscious domain contains a database $D_{f}$ of forbidden ideas. The comparator $\mathrm{COM}_{\mathrm{c}}$ measures not only the distance $\rho\left(J, D_{i}\right)$ between an idea-attractor $J$ (which has been transmitted to the conscious domain from the unconscious domain) and the set of interesting idea $D_{i}$, but also the distance $\rho\left(J, D_{i}\right)$ between an idea-attractor $J$ and the set of forbidden ideas $D_{f}:$

$$
\rho\left(J, D_{f}\right)=\min _{L \in D_{f}} \rho(J, L) .
$$

If $J$ is close to some forbidden idea $L_{0}$, then $\rho\left(J, D_{f}\right)$ is small. If $J$ is far from all forbidden ideas $L \in D_{f}$, then $\rho\left(J, D_{f}\right)$ is large.

We define a measure of interdiction $F(J)$ as

$$
F(J)=\frac{1}{1+\rho\left(J, D_{f}\right)} .
$$

$F(J)$ is large if $\rho\left(J, D_{f}\right)$ is small and $F(J)$ is small if $\rho\left(J, D_{f}\right)$ is large.

For the $m$-adic metric, $\rho\left(J_{1}, J_{2}\right) \leq 1$. Thus $F(J) \geq 1 / 2$. So $F$ takes values in the segment $[1 / 2,1]$. Here the sentence ' $F(J)$ is very small' means that $F(J) \approx 1 / 2$ and ' $F(J)$ is very large' means that $F(J) \approx 1$.

The control center $C C$ must take into account not only the level of interest $I(J)$ of an idea $J$ but also the level of interdiction $F(J)$ of an idea $J$. The struggle between interest $I(J)$ and interdiction $F(J)$ induces all essential features of human psychology. We consider a simple model of such a struggle. For an idea $J$, we define consistency (between interest and interdiction) as $T(J)=a I(J)-b F(J)$, where $a, b>0$ are some weights depending on a cognitive system $\tau$. Some $\tau$ could use more complex functionals for consistency. For example,

$$
T(J)=a I^{\alpha}(J)-b I^{\beta}(J)
$$

where $\alpha, \beta>0$, are some powers. There exists a threshold of realization $T_{\mathrm{rz}}$ such that if $T(J) \geq T_{\mathrm{rz}}$, then the idea $J$ is sent to the collector $Q$ for ideas waiting for realization. If $T(J)<T_{\mathrm{rz}}$, then the idea $J$ is deleted.

It is convenient to consider a special block in the conscious domain, analyzer, $\mathrm{AN}_{\mathrm{c}}$.

This block contains the comparator $\mathrm{COM}_{\mathrm{c}}$ which measures distances $\rho\left(J, D_{i}\right)$ and $\rho\left(J, D_{f}\right)$; a computation device which calculates measures of interest $I(J)$, interdiction $F(J)$ and consistency $T(J)$ and checks the condition $T(J) \geq T_{r z}$; a transmission device which sends $J$ to $Q$ or trash. The order in the queue $Q$ is based on the quantity $T(J)$. It is also convenient to introduce a block $\mathrm{SER}_{\mathrm{c}}$, server, in the conscious domain which orders ideas in $Q$ with respect to values of consistency $T(J)$.

We can again assume that there exists a threshold $T_{+}$such that ideas $J$ with $T(J) \geq T_{+}$must be realized in any case. This threshold plays the 
important role in the process of the time evolution of consistency $T(t, J)$ of an idea $J$ in $Q: T(t, J)=l\left(t-t_{0}\right) T(J)$, where the coefficient $l(t)$ decreases with increasing of $t$. Moreover, $T(t, J)=T(J)$ if $T(J) \geq T_{+}$. We note that $T_{+} \geq T_{\mathrm{rz}}$. It can be that interest and interdiction evolve in different ways: $I(t, J)=l_{i}\left(t-t_{0}\right) I(J)$ and $F(t, J)=l_{f}\left(t-t_{0}\right) F(J)$. Here $T(t, J)=$ $a I(t, J)-b F(t, J)$. Such a model is more realistic. A pessimist has quickly decreasing function $l_{i}(t)$ and slowly decreasing function $l_{f}(t)$. An optimist has slowly decreasing function $l_{i}(t)$ and quickly decreasing function $l_{f}(t)$.

Example 9.3. (Harmonic love). Let $\tau$ be a 'man' described by Model 3. The image $J_{0}$ of a woman $\gamma$ is transformed by $\pi_{\text {sex }}$ into the idea $J_{\text {love, } \gamma}$. Suppose that as in Example 1.2, $D_{i}$ contains $L_{\mathrm{blond}}$ and $\gamma$ is blond. However, $J_{\text {love }, \gamma}$ is not sent automatically to the queue of ideas waiting for realization. The idea $J_{\text {love, } \gamma}$ must be compared with the database $D_{f}$ of forbidden ideas. Suppose that the idea-image $G_{\text {tall }}=$ (tall woman) belongs to $D_{f}$. If $\gamma$ is tall, then $F\left(J_{\text {love }}\right)$ is quite large. The future of $J_{\text {love }}$ depends on the value of the consistency functional $T(J)$ (the relation between coefficients $a, b$ in (11) and values $I(J), F(J))$. However, this process still does not induce doubts or mental problems.

It seems that the consistency $T(J)$ does not determine the intensity of realization of $J$. An extremely interesting idea is not realized with strength that is proportional to the consistency magnitude $T(J)$. In fact, it is realized with strength that is proportional to the magnitude of interest $I(J)$. Moreover, larger interdiction also implies larger strength of realization. It seems natural to connect strength of realization with the quantity

$$
S(J)=c I(J)+d F(J)
$$

where $c, d>0$ are some parameters of the brain.

We call $S(J)$ strength of an idea $J$. In particular, $S(J)$ may play an important role in memory processes. We introduce a preserving threshold $S_{-}^{\mathrm{mem}}$ (compare with the preserving threshold $I_{-}^{\text {mem }}$ in model 2). The strength $S(t, J)$ of an idea $J$ in the working memory evolves as

$$
S(t, J)=l_{\mathrm{mem}}\left(t-t_{0}\right) S(J)
$$

where $l_{\text {mem }}$ is a decreasing function. If $S(t, J)<S_{-}^{\text {mem }}$, then at the instance of time $t$ the idea $J$ is deleted from working memory.

The structure of analyzer is given by Figure 4.

A cognitive system $\tau$ described by Model 3 has complex cognitive behavior. However, this complexity does not imply 'mental problems'. The use of consistency functional $T(J)$ solves the contradiction between interest and interdiction.

The main disadvantage of the cognitive system $\tau$ described by Model 3 is that the analyzer $\mathrm{AN}_{\mathrm{c}}$ permits the realization of ideas $J$ which have at the same time very high levels of interest and interdiction (if $I(J)$ and 


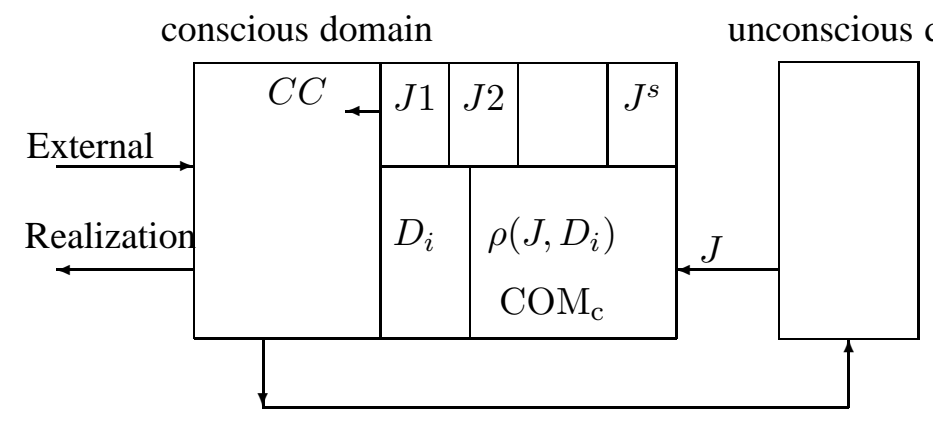

Figure 3: Model 2 of conscious/unconscious functioning (comparative analysis of ideas)

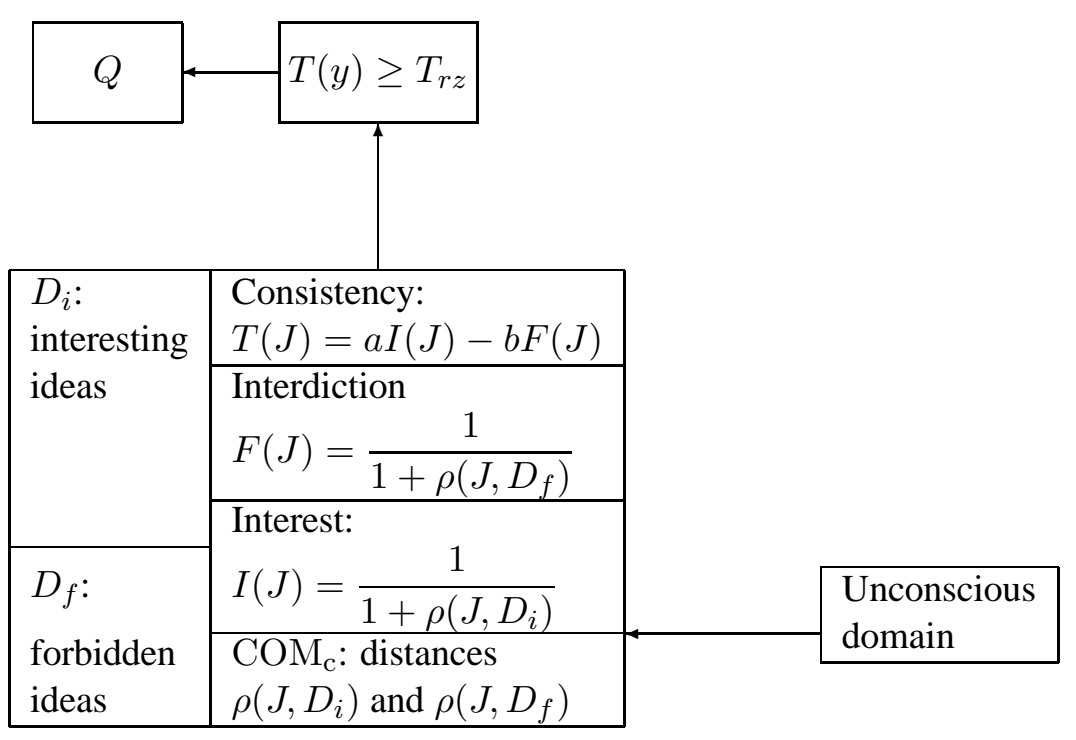

Figure 4: The structure of analyzer 
$F(J)$ compensate each other in the consistency function). For example, let $T(J)=I(J)-F(J)$. If the realization threshold $T_{\mathrm{rz}}=0$ the analyzer $\mathrm{AN}_{\mathrm{c}}$ sends to the collector $Q$ totally forbidden ideas $J$ (with $F(J) \approx 1$ ) having extremely high interest $(I(J) \approx 1)$.

Such a behavior ('a storm of cravings') can be dangerous (especially in a group of cognitive systems with a social structure). Therefore functioning of the analyzer $\mathrm{AN}_{\mathrm{c}}$ must be based on more complex analysis of $J$ which is not reduced to the calculation of $T(J)$ and testing $T(J) \geq T_{\mathrm{rz}}$.

\subsection{Model 4}

Suppose that a cognitive system described by Model 3 improves its brain by introducing two new thresholds $I_{\max }$ and $F_{\max }$. If $I(J) \geq I_{\max }$, then the idea $J$ is extremely interesting: $\tau$ can not simply delete $J$. If $F(J) \geq F_{\max }$, then an idea $J$ is strongly forbidden: $\tau$ can not simply send $J$ to $Q$.

If $J$ belongs to the 'domain of doubts'

$$
O_{d}=\left\{J: I(J) \geq I_{\max }\right\} \bigcap\left\{J: F(J) \geq F_{\max }\right\}
$$

the $\tau$ cannot take automatically (on the basis of the value of the consistency $T(J)$ ) the decision on realization of $J$.

Example 9.4. (Forbidden love). Let $\tau$ be a 'man' described by Model 4 . Here the image $J_{0}$ of a woman $\gamma$ contains not only the spatial image of $\gamma$, but also her social image. Suppose that the integral image $J_{0}$ is transformed by the thinking block $\pi_{\text {sex }}$ in the idea-attractor $J_{\text {love }}$. Suppose that, as in all previous examples, the image $L_{\text {blond }}$ belongs to $D_{i}$. Suppose that idea $G_{\mathrm{soc}}$ $=$ (low social level) belongs to $D_{f}$. Suppose that both $\rho\left(J_{\text {love }}, L_{\text {blond }}\right)$ and $\rho\left(J_{\text {love }}, G_{\text {soc }}\right)$ are very small. She is blond and poor! So $I\left(J_{\text {love }}\right) \geq I_{\max }$ (high attraction of the woman $\gamma$ for the $\tau$ ) and at the same time $F\left(J_{\text {love }}\right) \geq$ $F_{\max }$ (social restrictions are important for the $\tau$ ). In such a situation the $\tau$ cannot take any decision on the idea $J_{\text {love }}$.

\section{Hidden Forbidden Wishes, Psychoanalysis}

\subsection{Hidden forbidden wishes, idèe fixe}

On one hand, the creation of an additional block in analyzer $\mathrm{AN}_{\mathrm{c}}$ to perform $\left(I_{\max }, F_{\max }\right)$ analysis plays the positive role. Such a $\tau$ does not realize automatically (via condition $T(J) \geq T_{r z}$ ) dangerous ideas $J$, despite their high attraction. On the other hand, this step in the cognitive evolution induces hard mental problems for $\tau$. In fact, the appearance of the domain of doubts $O_{d}$ in the mental space is the origin of many psychical problems and mental diseases.

Let $\mathrm{AN}_{\mathrm{c}}$ find that idea $J$ belongs to $O_{d}$. The $\tau$ is afraid to realize $J$ as well as to delete $J$. The control center $C C$ tries to perform further analysis of 
such a $J$. $C C$ sends $J$ to the processing domain $\Pi$ as the initial problem for some processor $\pi^{1}$. If it produces an idea-attractor $J^{1}$ which does not belong to $O_{d}$, then the $\tau$ can continue normal cognitive functioning. However, if $\pi^{1}$ produces again an idea $J^{1}$ which belongs $O_{d}$, then $C C$ must continue the struggle against this doubtful idea. In the process of such a struggle $C C$ and some processors $\pi, \pi^{1}, \pi^{2}, \ldots$ are (at least partially) busy. An essential part of mental resources of $\tau$ is used not for reactions to external signals, but for the struggle with ideas $J$ belonging to $O_{d}$.

Typically this is a struggle with just one idèe fixe $J$, see Freud, 1933.

We can explain the origin of such an idèe fixe by our cognitive model. If an idea $J$ belonging to $O_{d}$ has been produced by the processor $\pi$, then it is natural that $C C$ will try again to use the same processor $\pi$ for analyzing the idea $J$. As $f_{\pi}(J)=J$ (the $J$ is a fixed point of the map $f_{\pi}$ ), then $\pi$ starting with $J$ will always produce the same idea $J$ (with the trivial sequence of iterations $J, J, \ldots, J)$. In general the doubtful idea $J$ can be modified by $C C$ (for example, on the basis of new information), $J \rightarrow J_{\text {mod }}$. An idea $J_{\bmod }$ can be considered as a perturbation of $J: \rho\left(J, J_{\text {mod }}\right)<s$, where $s$ is some constant. If $s>0$ is relatively small (so that $J_{\bmod }$ still belongs to the basis of attraction of $J)$, then iterations $J_{\mathrm{mod}}, J_{\bmod }^{1}=f_{\pi}\left(J_{\mathrm{mod}}\right), \ldots, J_{\bmod }^{N}=$ $f_{\pi}^{N}\left(J_{\text {mod }}\right), \ldots$ again converge to the $J$.

How can $C C$ stop this process of the permanent work with idèe fixe $J$ ?

The answer to this question was given in Freud, 1933: investigations of roots of hysterias and some other mental problems. By Freud idèe fixe $J$ is shackled by $C C$ into the unconscious domain.

In our model, the unconscious domain contains (besides the processing domain $\Pi$ and the unconscious control center $U C$ ) a special collector $D_{d}$ for doubtful ideas, forbidden wishes. After a few attempts to transform an idea $J$ belonging to the domain of doubtful ideas $O_{d}$ into some non-doubtful idea, $C C$ sends $J$ to $D_{d}$. We remark that the domain $O_{d}$ is a mental domain (a set of ideas) and $D_{d}$ is a 'hardware domain' (a set of chains of neurons used for saving of doubtful ideas).

What can we say about the further evolution of a doubtful idea $J$ in the collector $D_{d}$ ? It depends on a cognitive system $\tau$ (in particular, a human individual). In the 'purely normal case' the collector $D_{d}$ plays just the role of a churchyard for doubtful ideas. Such a $D_{d}$ has no output connections and idea $J$ will disappear after some period of time.

\subsection{Symptoms}

However, Freud demonstrated (on the basis of hundreds of cases) that advanced cognitive systems (such as human individuals) could not have 'purely normal behavior'. They could not perform the complete interment of doubtful ideas in $D_{d}$. 
In our model, the collector $D_{d}$ has an output connection with the unconscious control center $U C$. At this moment the existence of such a connection seems to be just a disadvantage in the mental architecture of $\tau$. It seems that such a cognitive system $\tau$ was simply not able to develop a physical system for $100 \%$-isolation of the collector $D_{d}$. However, later we shall demonstrate that the pathway

$$
C C \rightarrow D_{d} \rightarrow U C \rightarrow C C
$$

has important cognitive functions. In fact, such a connection was specially created in the process of evolution. But we start with the discussion on negative consequences of (12). Here we follow Freud, 1933.

In our mathematical model of Freud's theory of unconscious mind, an idea $J \in D_{d}$ is sent to $U C$. The unconscious control center $U C$ sends $J$ to one of the thinking processors $\xi$ in $\Pi$. $\xi$ performs iterations starting with $J$ as an initial idea. $\xi$ produces an idea-attractor $\tilde{J}=\lim _{N \rightarrow \infty} J_{N}, J_{0}=J$. In the simplest case $\xi$ sends the idea-attractor $\tilde{J}$ to the conscious domain. The $\mathrm{AN}_{\mathrm{c}}$ analyzes idea $\tilde{J}$. If $\tilde{J} \notin O_{d}$, then $\mathrm{AN}_{\mathrm{c}}$ sends $\tilde{J}$ to the collector $Q$ (of ideas waiting for realization). 8 After some period of waiting $\tilde{J}$ is sent to realization. 9 By such a realization $C C$ deletes $J$ from the collector $Q$. However, $C C$ does not delete the root of $\tilde{J}$, namely $J$, because $J$ is located in the unconscious domain and $C C$ is not able to control anything in this domain. The idea $\tilde{J}$ is nothing other than a performance of the forbidden wish $J$. Such unconscious transformations of forbidden wishes were studied by Freud (see Freud, 1933, for examples).

We note that if $U C$ sends a hidden forbidden wish $J$ to the same thinking processor $\pi$ which has already generated $J$ for $C C$, then (by the same reasons as in our previous considerations) $C C$ will again obtain the same doubtful idea $J$. Such a continuous reproduction of idèe fixe can take place. This is the root of obstinate doubtful wishes. This can imply mental deceases, because $C C$ could not stop the struggle with idèe fixe even by sending it to $D_{d}$. However, $U C$ may send $J$ to another thinking processor $\xi \neq \pi$. Here the idea-attractor $\tilde{J}$ (which has been produced starting with $J$ as the initial condition) differs from $J$. This is the real transformation of the forbidden wish. In general a new wish $\tilde{J}$ has no direct relation to the original wish $J$. This is nothing but a symptom of cognitive system $\tau$, Freud, 1933.

Starting with an initial idea $J_{0}$ a processor $\pi$ produces an attractor $J$; analyzer $\mathrm{AN}_{\mathrm{c}}$ computes quantities $I(J), F(J)$ (measures of interest and interdiction for the idea $J$ ); $\mathrm{AN}_{\mathrm{c}}$ considers $J$ as a doubtful idea: both measures of interest and interdiction are too high, $I(J) \geq I_{\max }, F(J) \geq F_{\max } ; \mathrm{AN}_{\mathrm{c}}$ sends $J$ to the collector of doubtful ideas $D_{d} ; J$ moves from $D_{d}$ to $U C ; U C$

\footnotetext{
${ }^{8}$ Of course, there may exist more complex pathways: $C C \rightarrow D_{d} \rightarrow U C \rightarrow \xi \rightarrow \xi^{1} \ldots \rightarrow$ $\xi^{m} \rightarrow U C \rightarrow \lambda \rightarrow \lambda^{1} \rightarrow \ldots \rightarrow \lambda^{k} \rightarrow \mathrm{AN}_{\mathrm{c}} \rightarrow \mathrm{Q} \rightarrow \mathrm{CC}$, where $\xi, \ldots, \xi^{m}, \lambda, \ldots, \lambda^{k}$ are some thinking processors.

${ }^{9}$ Of course, idea $\tilde{J}$ may be simply deleted in $Q$ if there are too many ideas in the queue and the strength $S(\tilde{J})$ of $\tilde{J}$ is not so large.
} 
sends it to some processor $\xi ; \xi$ produces an attractor $\tilde{J}$. Analyzer $\mathrm{AN}_{\mathrm{c}}$ can recognize $\tilde{J}$ as an idea which could be realized (depending on the distances $\rho\left(\tilde{J}, D_{i}\right)$ and $\left.\rho\left(\tilde{J}, D_{f}\right)\right)$ and send $\tilde{J}$ via the collector $Q$ to realization. This $\tilde{J}$ is a symptom induced by $J$ (in fact, by the initial idea $J_{0}$ ).

\subsection{Hysteric reactions}

In general a doubtful idea $J \in D_{d}$ is not only transferred into some symptom $\tilde{J}$, but it may essentially disturb functioning of the brain. Some thinking blocks $\pi_{\Pi}$ are directly connected to other thinking blocks. Suppose that, for example, the following pathway is realized: $J \in D_{d} \rightarrow U C \rightarrow \pi_{\Pi} \rightarrow \pi_{\mathrm{c}} \rightarrow$ $C C$. Suppose also that ideas $\lambda$ produced by $\pi_{\Pi}$ play the role of parameters for the block $\pi_{\mathrm{c}}: x_{n+1}=f_{\pi_{\mathrm{c}}}\left(x_{n}, \lambda\right)$. Let $C C$ obtain an image $J_{0}$ and send it to $\pi_{\mathrm{c}}$. However, instead of the normal value of the parameter $\lambda$, the $\pi_{\Pi}$ sends to $\pi_{\mathrm{c}}$ some abnormal value $\lambda_{\mathrm{ab}}$ induced by the hidden forbidden wish $J$. The $\pi_{\mathrm{c}}$ produces an attractor $L_{\mathrm{ab}}$ which may strongly differ from the attractor $L_{\text {norm }}$ corresponding to $\lambda_{\text {norm }}$, the value of the parameter produced by the processor $\pi_{\Pi}$ for the processor $\pi_{c}$ in the absence of the hidden forbidden wish $J$.

In such a way we explain, for example, hysteric reactions. A rather innocent initial stimulus $J_{0}$ can induce via interference with a doubtful idea $J \in D_{d}$ inadequate performance $L_{\mathrm{ab}}$. We can also explain why hidden forbidden wishes may induce physical diseases. Attempting to transform $J \in D_{d}$ into an idea which does not induce doubts and reflections, $U C$ can send $J$ to some thinking processor $\pi_{\text {phys }}$ that is responsible for some physical activity of $\tau$. We note that $U C$ considers $J$ as just a collection of mental states. This collection of mental states has different interpretations in different thinking systems. In particular, $J$ can correspond in $\pi_{\text {phys }}$ to some 'bad initial condition'. The corresponding attractor $L_{\mathrm{phys}}$ can paralyze the physical function ruled by $\pi_{\text {phys }}$.

\subsection{Feedback control based on doubtful ideas}

A cognitive system $\tau$ wants to prevent a new appearance of forbidden wishes $J$ (collected in $D_{d}$ ) in the conscious domain. The brain of $\tau$ has an additional analyzer $\mathrm{AN}_{\mathrm{d}}$ (located in the unconscious domain) that must analyze nearness of an idea attractor $L$ produced by some processor $\pi_{c}$ and ideas $J$ belonging to the collector of doubtful ideas $D_{d}$.

In our model, it is supposed that each hidden forbidden wish $J_{\mathrm{fd}}$ in the collector $D_{d}$ still remembers a thinking block $\pi$ which has produced $J_{\mathrm{fd}}$. This simply means that each idea $J_{\mathrm{fd}}$ in the $D_{d}$ has the label $\pi$. Thus $J_{\mathrm{fd}}=J_{\mathrm{fd}}(\pi) \in D_{d}$ is not just a collection of mental states. There is information that these states are related to the dynamical system $\pi$. The set of doubtful ideas $O$ which are collected in the collector $D_{d}$ can be split into subsets $O(\pi)$ of forbidden wishes corresponding to different thinking systems 
$\pi$. $\mathrm{AN}_{\mathrm{d}}$ contains a comparator $\mathrm{COM}_{\mathrm{d}}$ that measures the distance between an idea-attractor $J$ which has been produced by a thinking block $\pi$ and the set $O(\pi): \rho(J, O(\pi))=\min _{J_{\mathrm{fd}} \in O(\pi)} \rho\left(J, J_{\mathrm{fd}}\right)$. Then $\mathrm{AN}_{\mathrm{d}}$ calculates the measure of interdiction

$$
F_{d}(J)=\frac{1}{1+\rho(J, O(\pi))} .
$$

If $F_{d}(J)$ is large $(\approx 1)$, then an idea $J$ is too close to one of former hidden forbidden $\pi$-wishes. This idea should not be transmitted to the conscious domain.

Each individual $\tau$ has its own blocking threshold $F_{\mathrm{bl}}$ : if $F_{d}(J)<F_{\mathrm{bl}}$, then $J$ is transmitted; if $F_{d}(J) \geq F_{\mathrm{bl}}$, then $J$ is deleted. In the latter case $J$ will never come to the conscious domain 10 This threshold $F_{\mathrm{bl}}$ determines the degree of blocking of the thinking processor $\pi$ by forbidden wishes. For some individuals (having rather small values of $F_{\mathrm{bl}}$ ), a forbidden wish $J_{\mathrm{fd}}$ belonging to the set $O(\pi)$ may stop the flow of information from $\pi$ to the conscious domain. The same $J_{\mathrm{fd}}$ may play a negligible role for individuals having rather large magnitude of $F_{\mathrm{bl}}$. Therefore the blocking threshold $F_{\mathrm{bl}}$ is one of the important characteristics to distinguish normal and abnormal behaviors. We note that $F_{\mathrm{bl}}$ depends on a thinking block $\pi$ : $F_{\mathrm{bl}}=F_{\mathrm{bl}}(\pi)$. Thus the same individual $\tau$ can have the normal threshold for one thinking block $\pi$, relatively large $F_{\mathrm{bl}}(\pi)$, and abnormal degree of blocking for another thinking block $\pi^{\prime}$, relatively small $F_{\mathrm{bl}}\left(\pi^{\prime}\right)$.

Analyzer $\mathrm{AN}_{\mathrm{d}}$ computes the distance between the idea-attractor $J$ (produced by a thinking block $\pi$ ) and the domain $O(\pi)$ of hidden forbidden $\pi$ wishes. If this distance is relatively small, i.e., the measure of interdiction $F_{d}(J)$ is relatively large, then $J$ does not go to the conscious domain.

\section{Consequences for neurophysiology, neuroin- formatics and cognitive sciences}

\subsection{Hierarchical models of cognition}

Such models (especially for the visual system) were discussed in a number of works: Hubel and Wiesel, 1962, Bechtel and Abrahamsen, 1991, Ivanitsky, 1999, Watt and Phillips, 2000, Stringer and Rolls, 2002, Khrennikov, 1997, 1998a,b, 1999a,b, 2000a, b; Albeverio et al., 1999; Dubischar et al, 1999, Voronkov, 2002a, b, Sergin, 2007.

However, the hierarchical approach has not yet become commonly accepted in neurophysiology, neuroinformatics and cognitive sciences. Our mathematical model is fundamentally hierarchical. We were able to encode

\footnotetext{
${ }^{10}$ Analysis in the conscious domain could demonstrate that $T(J) \geq T_{\mathrm{rz}}$ and $I(J)<$ $I_{\max }, F(J)<F_{\max }$. In the absence of hidden forbidden wishes $J$ would be realized.
} 


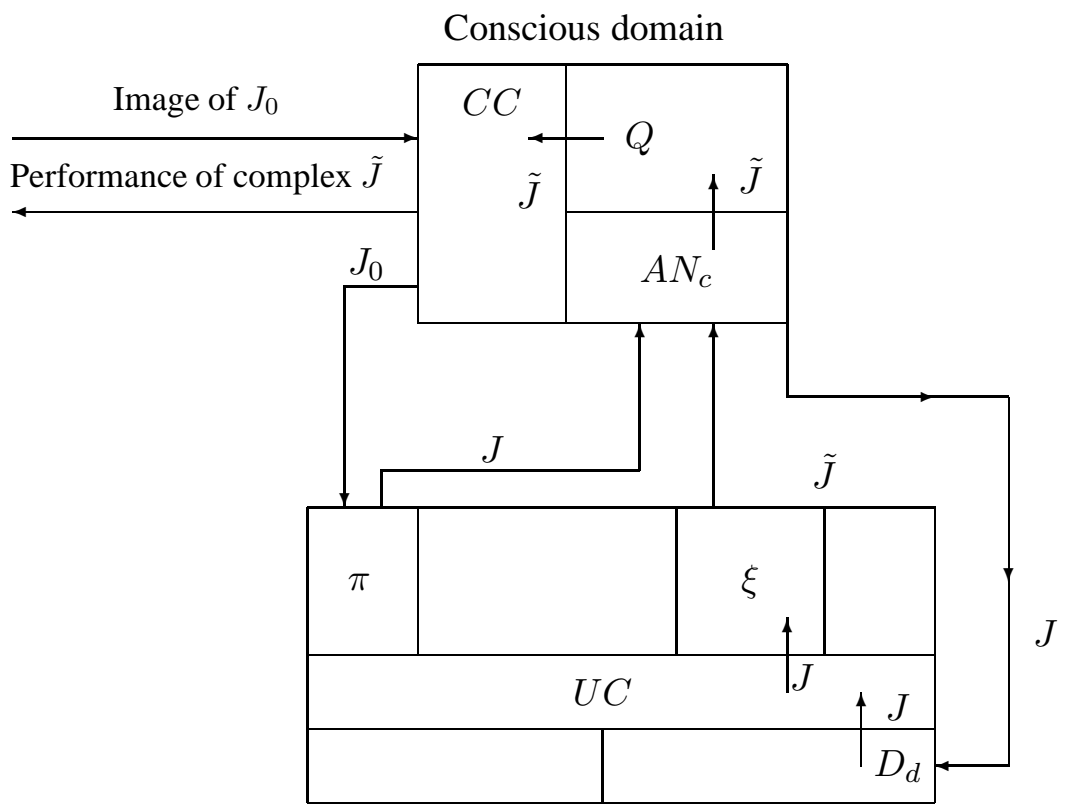

Unconscious domain

Figure 5: Symptom induced by a forbidden wish

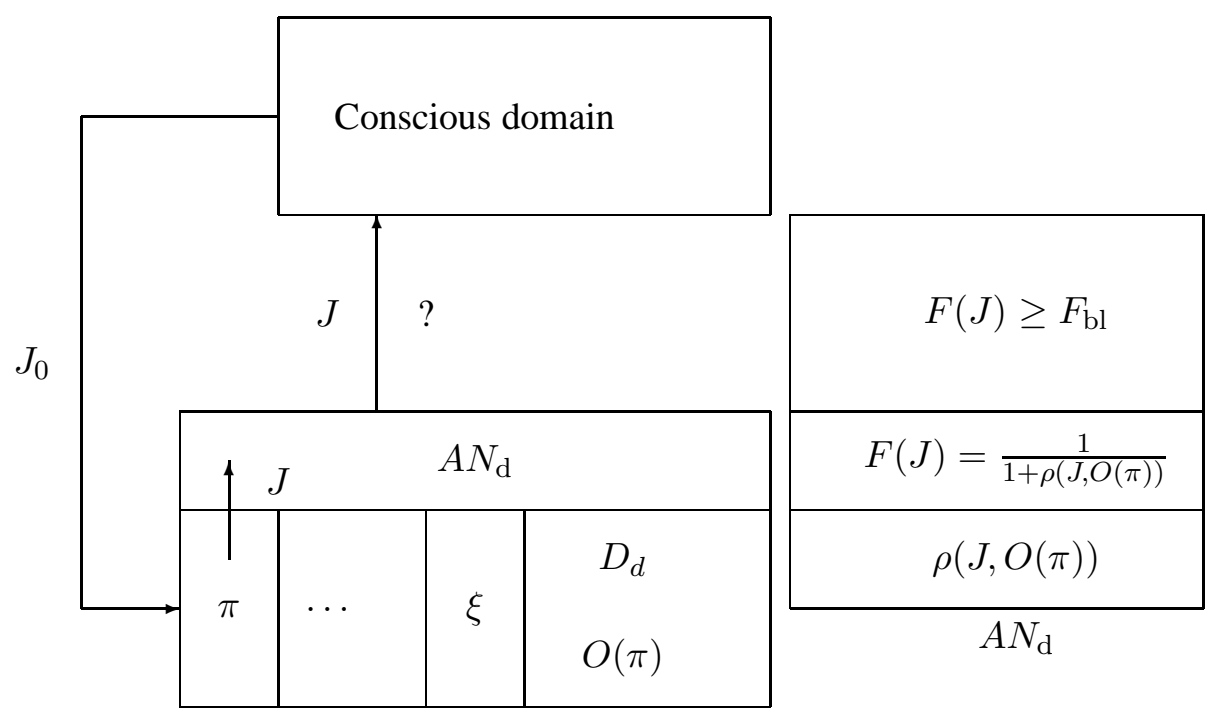

Unconscious domain

Figure 6: Interference of an idea-attractor with the domain of hidden forbidden wishes. Internal structure of the analyzer $\mathrm{AN}_{\mathrm{d}}$. 
hierarchy (both neuronal and mental) into the ultrametric geometry. Creation of a simple model of hierarchical mental space (which has natural coupling with the neuronal structure of the brain) provides a mathematical basis of the hierarchical approach to brain's functioning.

On the one hand, our model needs further justification from experimental neurophysiology. Unfortunately, at the moment there is no general consensus on the presence of hierarchical neuronal trees in the brain. The experimental research is characterised by diversity of opinions. Mathematical self-consistency of our hierarchical model might become a good stimulus for further research in neurophysiology, neuroinformatics and cognitive sciences to study the hierarchy of the brain, its functioning, cognition.

On the other hand, there was created a number of theoretical hierarchical models for processing of information in the brain, . However, these models were presented not on the level of mathematical modeling. It might be possible to use our mathematical formalism to present the mentioned approaches on the mathematical level.

\subsection{The problem of localization of mental functions}

This problem has been the source of permanent discussions in neurophysiology, neuroinformatics and cognitive sciences for more than one hundred years, see, e.g., Damasio, 2005, for discussions and references. Our mathematical model combines peacefully the views of both parties: the adherents of the localization hypothesis as well as the adherents of the non-localization hypothesis.

On the one hand, in our model each mental function $f$ is distributed over a neuronal tree. Branches of this tree go through different domains of the brain. Moreover, branches can contain even neurons belonging the spinal cord. Thus a branch can go from the cerebellum to the pons and then to the medulla oblongata and finally through the spinal cord to the conus medullaris. It is natural to include not only neurons, but also sensory receptors into neuronal trees (as belonging to the lowest level of hierarchical neuronal trees). In such a model delocalization of a mental function increases essentially.

Thus in our model not only the brain, but even the body participates in the thinking process.

On the other hand, the hierarchical structure of our model provides a rather sharp localization of a mental function $f$ (as a consequence of associative processing). The state $\alpha_{0}$ of the root-neuron plays the crucial role. The states of neurons of the fist level are less important and so on.

We proposed the distributed model of processing of information in the brain with hierarchical localization. 


\subsection{Binding problem}

This problem is also well known in neurophysiology, neuroinformatics and cognitive sciences, see, e.g., Revonsuo and Newman, 1999, Thiele and Stoner, 2003, Zimmer et al, 2006, for discussions and possible solutions. In our approach the binding problem was solved on the morphological level. The fundamental units of information processing in the brain are not single neurons (or even localized neuronal populations), but hierarchical neuronal trajectories. The presence of the hierarchical structure on these neuronal trajectories solves some problems of binding, e.g., the problem of consistency of time scales. By operating with associations determined by short initial segments of hierarchical neuronal trajectories (and not with detailed mental images determined by the whole hierarchical neuronal trajectories) the brain uses only the top part of a neuronal tree. Such a computational architecture minimizes essentially the time of processing, cf. applications to image recognition and compression, Benois-Pineau et. al, 2001, Khrennikov and Kotovich, 2002, Khrennikov, Kotovich, and Borzistaya, 2004.

\subsection{The problem of invariance of mental images}

How can my brain recognize the image of my lovely woman? She can be dressed in different ways, she can express totally different emotions (from great pleasure to terrible scandals ), and so on. In our model the invariance is achieved through using the association-representation. Only the states of neurons belonging to the top levels of a neuronal tree (which is responsible for for the image of my lovely woman) are important in recognition. Temporary differences are represented by lower levels, cf. with $m$-adic image recognition algorithms Benois-Pineau et. al, 2001, Khrennikov and Kotovich, 2002, Khrennikov, Kotovich, and Borzistaya, 2004.

\section{Consequences for psychology and neuropsy- chology}

Our model gives the possibility to perform mathematical simulation of psychological behaviour. We performed geometrization of psychology, geometropsychology. By introducing a mathematical model of mental space we incorporated psychology into the same rigorous mathematical framework as it was done in physics by Newton and Hamilton. The crucial point is that geometries of physical and mental spaces differ very much. The presence of the rigid hierarchical structure plays the fundamental role in the $m$-adic mathematical model of mental space.

Hierarchical representations are well accepted in psychology. Our model provides the corresponding mathematical basis. 
By coupling the $m$-adic mental space with neuronal trees we constructed a bridge between neurophysiology and psychology. Thus our model can be considered as a contribution to neuropsychology.

We applied the $m$-adic mental model to mathematical modeling of Freud's psychoanalysis. We aware about diversity of views on Freud's psychoanalysis in modern psychology, see, e.g., Macmillan, 1997, Gay, 1988, YoungBruehl, 1998 as well as Stein et al., 2006, Solms, M., 2006a, 2006b, for debates. Our model supports the view which was presented in the journal "Neuro-psychoanalysis:"

It would be possible to create an ongoing dialogue with the aim of reconciling psychoanalytic and neuroscientific perspectives on the mind. This goal is based on the assumption that these two historically divided disciplines are ultimately pursuing the same task, namely, "attempt[ing] to make the complications of mental functioning intelligible by dissecting the function and assigning its different constituents to different component parts of the [mental] apparatus," Freud, 1900, p. 536. Notwithstanding the fact that psychoanalysis and neuroscience have approached this important scientific task from radically different perspectives, the underlying unity of purpose has become increasingly evident in recent years as neuroscientists have begun to investigate those "complications of mental functioning" that were traditionally the preserve of psychoanalysts. This has produced an explosion of new insights into problems of vital interest to psychoanalysis, but these insights have not been reconciled with existing psychoanalytic theories and models.

We can complete this manifest by the remark that neurophysiology has an essentially higher level of the mathematical representation than traditional psychoanalysis. Therefore coupling of psychoanalysis with neurophysiology provides new perspectives in mathematization of psychoanalysis. Our $m$-adic model serves precisely to such a purpose. Starting with a model of the neuronal structure of the brain, hierarchical neuronal trees, we created the $m$-adic model of mental space. This model was then applied to mathematical modeling of psychoanalysis. The $m$-adic distance on mental space is the basis of forming of measures of interest and interdiction and consequently hidden forbidden wishes and, finally, symptoms and hysteries. And this $m$-adic distance on mental space is induced by the neuronal structures hierarchical neuronal trees. There would be no psychical problems without mental hierarchy in the brain. Psychical problems is the price for advantages of hierarchical processing of information in the brain.

\section{Possible consequences for medicine/psychiatry}

Our model provides an interesting explanation of differences in psychical consequences of the same events and mental experiences. Already Freud pointed our to the role of such differences in forming of symptoms. The same mental experience could play a minor role and it would be immediately 
forgotten by one person and it could be the starting point a hard psychical illness for another person, Freud, 1933.

In our model this differences in psychical reactions to the same mental experience are explained by the scheme of mental architecture which is based on blocking thresholds (such a threshold model is of course based on the presence of the ultrametric structure on the mental space). If this hypothesis were confirmed by clinical investigations, there will be opened new ways for mental treatment. There can be developed both chemical and psychoanalytic methods for changing the magnitudes of blocking thresholds. To develop chemical methods of treatment, we should find the neurophysiological basis of blocking thresholds. There can be also developed psychoanalytic methods for changing the magnitudes of blocking thresholds. By special training patients can learn to operate with blocking thresholds of lower or higher magnitudes. If a patient were able to make his blocking thresholds smaller, some hidden forbidden wishes would come to the consciousness. On the one hand, his conscious mental life would become essentially more complicated. On the other hand, some symptoms would disappear. If a patient were able to make his blocking thresholds larger, his mental (and in particular, emotional) behavior would become more plane. It could be important for treatment of patients with aggressive and destructive behavior. Such learning procedures could be based on the brain-computer interface approach.

\section{Possible consequences for artificial life}

Investigations on artificial intelligence are oriented mainly to creation of artificial systems for motion in physical space and performing various tasks in this space, e.g., creation of robots 11 Our $m$-adic hierarchical model provides possibility for simulation of human psychology. In principle, on the basis of our model artificial intellectual systems can be created. They would live rich emotional life: numerous interesting ideas, constraints, forbidden ideas, hidden forbidden wishes, feedback control based on them, symptoms and finally psychical problems, including hysteries. Such artificial intellectual systems would be able to love (of course, only at the mental level), they could have various psychical illnesses. How can we use such psychological robots?

We can test on such psychological robots different models of mental architecture, for example, our hypothesis on blocking thresholds as well as our general hierarchical model. Creation of populations of psychological robots gives the possibility for simulation of complex socio-psychological life.

Conclusion. A mathematical model for hierarchical encoding of mental information was created. Mental space (a mental analog of physical space) is realized as an m-adic tree. Processing of mental information is realized by

\footnotetext{
${ }^{11}$ Of course, artificial intelligence activity is not restricted to robots. We can mention creation of chess playing machines.
} 
dynamical systems on such a tree. Interplay between unconscious and conscious information flows generates interesting psychological behavior. Consequences for neurophysiology, neuroinformatics, and cognitive sciences as well as for psychology and neuropsychology, and even medicine/psychiatry and artificial ("psychological robots") were discussed. The m-adic hierarchical model of processing of mental information plays the role of the unifying mathematical basis for a number of various neurophysiological, neuroinformatical and cognitive models of brain's functioning.

\section{REFERENCES}

Albeverio, S., Khrennikov, A. Yu., Kloeden, P., 1999. Memory retrieval as a $p$-adic dynamical system. Biosystems 49, 105-115.

Amit, D., 1989. Modeling Brain Function. Cambridge Univ. Press, Cambridge.

Ashby, R., 1952, Design of a brain. Chapman-Hall, London.

Baars, B. J., 1997, In the theater of consciousness. The workspace of mind. Oxford University Press, Oxford.

Bar, M., 2005. Top-down facilitation of visual object recognition. In Neurobiology of attention. Eds.: L. Itti, G. Rees, and J. K. Tsotsos, Elsevier, Amsterdam, 140-145.

Bechtel, W., Abrahamsen, A., 1991. Connectionism and the mind. Basil Blackwell, Cambridge.

Bechterew, W., 1911. Die Funktionen der Nervencentra. Jena, Fischer.

Benois-Pineau, J., Khrennikov, A. Yu., and Kotovich, N. V., 2001. Segmentation of images in $p$-adic and Euclidean metrics. Dokl. Akad. Nauk. 381, N. 5, 604-609. English Translation: Doklady Mathematics 64, N. 3, 450-455.

Blakemore, S. J., Decety, J., 2001. From the perception of action to the understanding of intention. Nature Reviews Neuroscience 2 (8), 561-567.

Blomberg, C., Liljenstrm, H., Lindahl, B.I.B., and Arhem, P., (Eds), 1994.Mind and Matter: Essays from Biology, Physics and Philosophy: An Introduction, J.theor. Biol. 171.

Bredenkamp, J. (1993). Die Verknupfung verschiedener Invarianzhypothesen im Bereich der Gedachtnispsychologie. Zeitschrift fur Experimentelle und Angewandte Psychologie, 40, 368-385.

Chaminade, T., Meary, D., Orliaguet, J.P., Decety, J., 2001. Is perceptual anticipation a motor simulation? A PET study Neuroreport 12 (17), 36693674.

Chomsky, N., 1963, Formal properties of grammas. Handbook of mathematical psychology. Luce, R. D.; Bush, R.R.; Galanter, E.; Eds. 2, Wiley: New York, pp. 323-418.

Churchland, P.S., Sejnovski, T., 1992, The computational brain. MITP: Cambridge.

Clark, A., 1980. Psychological models and neural mechanisms. An examination of reductionism in psychology. Clarendon Press, Oxford. 
Conte, E., Pierri, G.P., Mendolicchio, L., Federici, A., Zbilut J.P., 2006. A quantum model of consciousness interfaced with a non-Lipschitz chaotic dynamics of neural activity. Chaos, Solitons and Fractals.

Damasio, H. and Damasio, A. R., 1989. Lesion analysis in neuropsychology. New-York, Oxford Univ. Press.

Damasio, A. R., 2005. Descartes' error: emotion, reason, and the human brain. Penguin (Non-Classics).

Dragovich, B., and Dragovich, A., 2006. A p-Adic model of DNA sequence and genetic code. Electronic preprint: q-bio.GN/0607018

Dubischar D., Gundlach, V. M., Steinkamp, O., Khrennikov, A. Yu., 1999, A $p$-adic model for the process of thinking disturbed by physiological and information noise. J. Theor. Biology 197, 451-467.

Edelman, G. M. 1989. The remembered present: a biological theory of consciousness. New York, Basic Books.

Eliasmith, C., 1996. The third contender: a critical examination of the dynamicist theory of cognition. Phil. Psychology 9(4), 441-463.

Fodor, J.A. and Pylyshyn, Z. W., 1988. Connectionism and cognitive architecture: a critical analysis, Cognition, 280, 3-17.

Freud, S, 1900. The interpretation of dreams. Standard Edition, 4 and 5.

Freud, S., 1933. New introductory lectures on psychoanalysis. New York, Norton.

Frith, C.D., Frith, U., 1999, Cognitive psychology - Interacting minds A biological basis. Science 286 (5445), 1692-1695.

Fuster, J.M.D., 1997. The prefrontal cortex: anatomy, physiology, and neuropsychology of the frontal lobe. Philadelphia, Lippincott-Raven.

Gay, P., 1988. Freud: A life for our time. W.W. Norton, NY.

Geissler, H.-G., Klix, F., and Scheidereiter, U. (1978). Visual recognition of serial structure: Evidence of a two-stage scanning model. In E. L. J. Leeuwenberg and H. F. J. M. Buffart (Eds.), Formal theories of perception (pp. 299-314). Chichester: John Wiley.

Geissler, H.-G. and Puffe, M. (1982). Item recognition and no end: Representation format and processing strategies. In H.-G. Geissler and Petzold (Eds.), Psychophysical judgment and the process of perception (pp. 270281). Amsterdam: North-Holland.

Geissler, H.-G. (1983). The Inferential Basis of Classification: From perceptual to memory code systems. Part 1: Theory. In H.-G. Geissler, H. F. Buffart, E. L. Leeuwenberg, and V. Sarris (Eds.), Modern issues in perception (pp. 87-105). Amsterdam: North-Holland.

Geissler, H.-G. (1985). Zeitquantenhypothese zur Struktur ultraschneller Gedachtnisprozesse. Zeitschrift fur Psychologie, 193, 347-362. Geissler, H.-G. (1985b). Sources of seeming redundancy in temporally quantized information processing. In G. d'Ydewalle (Ed.), Proceedings of the XXIII International Congress of Psychology of the I.U.Psy.S., Volume 3 (pp. 199228). Amsterdam: North-Holland. 
Geissler, H.-G. (1987). The temporal architecture of central information processing: Evidence for a tentative time-quantum model. Psychological Research, 49, 99-106. Geissler, H.-G. (1990). Foundations of quantized processing. In H.-G. Geissler (Ed.), Psychophysical explorations of mental structures (pp. 193-210). Gottingen, Germany: Hogrefe and Huber Publishers.

Geissler, H.-G. (1992). New magic numbers in mental activity: On a taxonomic system for critical time periods. In H.-G. Geissler, S. W. Link, and J. T. Townsend (Eds.): Cognition, information processing and psychophysics (pp. 293-321). Hillsdale, NJ: Erlbaum.

Geissler, H.-G. and Kompass, R. (1999). Psychophysical time units and the band structure of brain oscillations. 15th Annual Meeting of the International Society for Psychophysics, 7-12.

Geissler, H.-G., Schebera, F.-U., and Kompass, R. (1999). Ultra-precise quantal timing: Evidence from simultaneity thresholds in long-range apparent movement. Perception and Psychophysics, 6, 707-726.

Geissler, H.-G., and Kompass, R. (2001). Temporal constraints in binding? Evidence from quantal state transitions in perception. Visual Cognition, 8, 679-696.

Hopfield, J. J., 1982. Neural networks and physical systems with emergent collective computational abilities. Proc. Natl. Acad. Sci. USA 79, 1554-2558.

Hoppensteadt, F. C., 1997. An introduction to the mathematics of neurons: modeling in the frequency domain. Cambridge Univ. Press, New York.

Hubel, D., and Wiesel, T., 1962. Receptive fields, binocular interaction and functional architecture in the cat's visula cortex. J. Physiol. 160, 106154.

Ivanitsky, A.M., 1999. Brain's physiology and the origin of the human's subjective world. J. of High Nerves Activity 49, N. 5, 707-713.

Khrennikov, A. Yu. , 1997. Non-Archimedean analysis: quantum paradoxes, dynamical systems and biological models. Kluwer, Dordrecht.

Khrennikov, A. Yu., 1998a. Human subconscious as the $p$-adic dynamical system. J. of Theor. Biology 193, 179-196.

Khrennikov, A. Yu., 1998b. p-adic dynamical systems: description of concurrent struggle in biological population with limited growth. Dokl. Akad. Nauk 361, 752.

Khrennikov, A. Yu., 1999a, Description of the operation of the human subconscious by means of $p$-adic dynamical systems. Dokl. Akad. Nauk 365, 458-460.

Khrennikov, A. Yu., 2000a, $p$-adic discrete dynamical systems and collective behaviour of information states in cognitive models. Discrete Dynamics in Nature and Society 5, 59-69.

Khrennikov, A. Yu., 2000b. Classical and quantum mechanics on $p$-adic trees of ideas. BioSystems 56, 95-120. 
Khrennikov A.Yu., 2002. Classical and quantum mental models and Freud's theory of unconscious mind. Series Math. Modelling in Phys., Engineering and Cognitive sciences, 1. Växjö Univ. Press, Växjö.

Khrennikov, A. Yu., and Kotovich, N.V., 2002. Representation and compression of images with the aid of the $m$-adic coordinate system. Dokl. Akad. Nauk. 387 N. 2, 159-163.

Khrennikov, A.Yu., 2004a, Information dynamics in cognitive, psychological, social, and anomalous phenomena. Kluwer, Dordreht.

Khrennikov, A. Yu., 2004b, Probabilistic pathway representation of cognitive information. J. Theor. Biology 231, 597-613.

Khrennikov, A. Yu., Kotovich, N. V., Borzistaya, E. L., 2004. Compression of images with the aid of representation by $p$-adic maps and approximation by Mahler's polynomials. Dokl. Akad. Nauk 396, N 3, 305-308. English Translation: Doklady Mathematics 69 N 3, 373-377.

Klix, F., and van der Meer, E. (1978). Analogical reasoning - an approach to mechanisms underlying human intelligence performances. In F. Klix (Ed.), Human and artificial Intelligence (p. 212). Berlin: Deutscher Verlag der Wissenschaften.

Kristofferson, M. W. (1972). Effects of practice on character-classification performance. Canadian Journal of Psychology, 26, 540-560.

Kristofferson, A. B. (1980). A quantal step function in duration discrimination. Perception and Psychophysics, 27, 300-306.

Kristofferson, A. B. (1990). Timing mechanisms and the threshold for duration. In Geissler, H.-G. (Ed., in collaboration with M. H. Muller and W. Prinz), Psychophysical explorations of mental structures (pp. 269-277). Toronto: Hogrefe and Huber Publishers.

Luczak, A., Bartho, P., Marguet, S. L., Buzsaki, G., Hariis, K.D, 2007, Neocortical spontaneous activity in vivo: cellular heterogeneity and sequential structure. Preprint of CMBN, Rutgers University.

Macmillan, M., 1997. The Completed Arc: Freud Evaluated. MIT Press., Cambridge MA.

Murtagh, 2004. On ultrametricity, data coding, and computation. Journal of Classification 21, 167-184.

Oztop, E., Wolpert, D., Kawato, M. 2005, Mental state inference using visual control parameters. Cognitive Brain Research 22 (2), 129-151.

Pitkänen, M., 1998. TGD inspired theory of consiousness with applications to biosystems. Electronic book: http://www.physics.helsinki.fi/ matpitka/cbookI.html

Pitkänen, M. 2006. Could genetic code be understood number theoretically? Electronic preprint: www.helsinki.fi/matpitka/pdfpool/genenumber.pdf.

Revonsuo, A and Newman, J., 1999. Binding and Consciousness. Consciousness and Cognition 8, 123-127.

Sergin, V. Ja., 2007. Biologically reasonable model of visual perception: hierarchy of unifying sensor characteristics. In Neuroinformatics-2007, Lectures on Neuroinformatics, part 2. Ed.: Yu. V. Tumenzev, Moscow Institute of Engineering and Physics Press, Moscow, 77-120. 
Smythies, J.R., 1970. Brain mechanisms and behaviour. Blackwell Sc. Publ.: Oxford.

Solms, M., 2002. An Introduction to the Neuroscientific Works of Freud. In The Pre-Psychoanalytic Writings of Sigmund Freud. Eds.: G. van der Vijver and F. Geerardyn. Karnac, London, 25-26.

Solms, M., 2006a. Putting the psyche into neuropsychology. Psychologist 19 (9), 538-539.

Solms, M., 2006b. Sigmund Freud today. Psychoanalysis and neuroscience in dialogue. Psyche-Zeiteschrift fur Psychoanalyse und ihre Anwendungen 60 (9-10), 829-859.

Stein, D. J., Solms, M., van Honk, J., 2006. The cognitive-affective neuroscience of the unconscious. CNS Spectrums 11 (8), 580-583.

Stringer, S.M., and Rolls, E., 2002. Invariant object recognition in the visual system with novel views of 3D objests. Neural. Comput. 14, 25852596.

Strogatz, S. H., 1994. Nonlinear dynamics and chaos with applications to physics, biology, chemistry, and engineering. Addison Wesley, Reading, Mass.

Teghtsoonian, R. (1971): On the exponents in Stevens' law and on the constant in Ekman's law. Psychological Review, 78, 71 - 80.

Thiele, A., and Stoner, G., 2003. Neuronal synchrony does not correlate with motion coherence in cortical area MT. Nature 421, 366-370.

van Gelder, T., Port, R., 1995. It's about time: Overview of the dynamical approach to cognition. in Mind as motion: Explorations in the dynamics of cognition. Ed.: T. van Gelder, R. Port. MITP, Cambridge, Mass, 1-43.

van Gelder, T., 1995. What might cognition be, if not computation? J. of Philosophy 91, 345-381.

Vladimirov, V. S., Volovich, I. V., and Zelenov, E. I., 1994, p-adic Analysis and Mathematical Physics. World Scientific Publ., Singapore.

Voronkov, G.S., 2002a. Information and brain: viewpoint of neurophysiolog. Neurocomputers: development and applications N 1-2, 79-88.

Voronkov, G.S., 2002b. Why is the perceived visual world non-mirror? Int. J. of Psychophysiology 34, 124-131.

Watt, R.J., and Phillips, W.A., 2000. The function of dynamical grouping in vision. Trends Cogn. Sc. 4, 447-454.

Young-Bruehl, E., 1998. Subject to Biography. Harvard University Press, Boston.

Zimmer, H., Mecklinger, A., and Lindenberger U., 2006. Handbook of binding and memory. Perspectives from cognitive neuroscience. Oxford Univ. Press, Oxford. 